\title{
A comparative study: the prospective influence of nanovectors in leveraging the chemopreventive potential of COX-2 inhibitors against skin cancer
}

This article was published in the following Dove Press journal: International Journal of Nanomedicine

\author{
Noha Khalifa Abo Aasy (D) \\ Doaa Ragab ${ }^{1,2}$ \\ Marwa Ahmed Sallam ${ }^{1,3}$ \\ Doaa A Abdelmonsif ${ }^{4,5}$ \\ Rania G Aly ${ }^{6}$ \\ Kadria A Elkhodairy' \\ 'Department of Industrial Pharmacy, \\ Faculty of Pharmacy, Alexandria \\ University, Alexandria 21521, Egypt; \\ ${ }^{2}$ Department of Chemical and \\ Biochemical Engineering, Faculty of \\ Engineering, University of Western \\ Ontario, London, Ontario, Canada; ${ }^{3}$ John \\ A. Paulson School of Engineering and \\ Applied Sciences, Harvard University, \\ Cambridge, MA, USA; ${ }^{4}$ Department of \\ Medical Biochemistry, Faculty of \\ Medicine, Alexandria University, \\ Alexandria, Egypt; ${ }^{5}$ Molecular Biology and \\ Nanomedicine Labs, Centre of \\ Excellence for Regenerative Medicine \\ Research \& Applications, University of \\ Alexandria, Alexandria, Egypt; \\ ${ }^{6}$ Department of Surgical Pathology, \\ Faculty of Medicine, Alexandria \\ University, Alexandria, Egypt
}

\begin{abstract}
Introduction: This study was conducted to elucidate the chemopreventive potential, cytotoxic, and suppression of cellular metastatic activity of etodolac (ETD)-loaded nanocarriers. Methods: To esteem the effect of charge and composition of the nanovectors on their performance, four types of vectors namely, negative lipid nanovesicles; phosalosomes (N-Phsoms), positive phosalosomes (P-Phsoms), nanostructured lipid carriers (NLCs) and polymeric alginate polymer (AlgNPs) were prepared and compared. ETD was used as a model cyclo-oxygenase-2 (COX-2) inhibitor to evaluate the potency of these nanovectors to increase ETD permeation and retention through human skin and cytotoxicity against squamous cell carcinoma cell line (SCC). Moreover, the chemopreventive activity of ETD nanovector on mice skin cancer model was evaluated.
\end{abstract}

Results: Among the utilized nanovectors, ETD-loaded N-Phsoms depicted spherical vesicles with the smallest particle size $(202.96 \pm 2.37 \mathrm{~nm})$ and a high zeta potential of $-24.8 \pm 4.16 \mathrm{mV}$. N-Phsoms exhibited 1.5, and 3.6 folds increase in the ETD amount deposited in stratum corneum, epidermis and dermis. Moreover, cytotoxicity studies revealed a significant cytotoxic potential of such nanovector with $\mathrm{IC}_{50}=181.76$ compared to free $\mathrm{ETD}\left(\mathrm{IC}_{50}=982.75\right)$, correlated to enhanced cellular internalization. Its efficacy extended to a reduction in the relative tumor weight with 1.70 and 1.51-fold compared to positive control and free ETD, that manifested by a 1.72-fold reduction in both COX-2 and proliferating cell nuclear antigen mRNA (PCNA-mRNA) levels and 2.63-fold elevation in caspase-3 level in skin tumors relative to the positive control group with no hepato-and nephrotoxicity.

Conclusion: Encapsulation of ETD in nanovector enhances its in-vitro and in-vivo antitumor activity and opens the door for encapsulation of more relevant drugs.

Keywords: COX-2, confocal laser microscopy, skin cancer, metastasis, cytotoxicity, chemoprevention

\section{Introduction}

Skin cancer (SC) is one of the most common form of malignancy, accounting for $4.5 \%$ of cancer cases, but it is not the deadliest type. More than 3.5 million cases of non-melanomatous skin cancer (NMSC) reported annually with an average of one million case annually evolved. The most common forms of SC are NMSC, which includes both basal cell carcinoma (BCC) and squamous cell carcinoma (SCC). Excessive exposure to ultraviolet (UV) ray is the main factor of transformation of normal skin cells to SC cells. Cytotoxicity of the conventional chemotherapeutic agents to both cancerous and normal skin cells provoked the need of more effective treatment with less side effects. ${ }^{1-3}$
Correspondence: Noha Khalifa Abo Aasy Department of Industrial Pharmacy, Faculty of Pharmacy, Alexandria University, I-Azarita Square, Alexandria, Egypt

Tel +20 I22 710 1058

$\mathrm{Fax}+2034873273$

Email drnohakhalifa@yahoo.com 
Various studies have elucidated the role of cyclo-oxygenase-2 (COX-2) in the promotion and poor prognosis of various types of cancer. It is suggested that COX-2 has a significant correlation to the overexpression of vascular endothelial growth factor c (VEGF-C), which contributes to the metastasis of various types of cancer such as oral, gastric, breast, lung, colon, head, and neck cancers. Accordingly, COX-2-selective inhibitors have been shown to induce apoptosis in wide variety of solid tumor cell lines, such as glioma, head and neck, cervical, and colon cancer. ${ }^{4-7}$

Noteworthy, COX-2 inhibitors intake as a single therapy showed a chemoprotective potential against mouse skin carcinogenesis, breast, and colon cancer. Moreover, co-administration of selective COX-2 inhibitors with chemotherapeutic agents has been shown to enhance the effects of the chemotherapeutic agent in human colon, pancreas, and lung cancer cells. ${ }^{4,8-12}$

COX-2 inhibitors may also serve as chemosensitizers, promoting the efficacy of chemotherapeutic agents by overcoming its cancerous cell resistance. In ovarian cancer, COX-2 and multidrug resistance 1 (MDR1) gene expression levels are associated with a chemotherapeutic drug resistance and poor prognosis. COX-2 inhibition was found to induce downregulation in MDR1m-RNA expression. Therefore, combinational therapy of both a selective COX-2 inhibitor and anticancer drug may improve the efficacy of the chemotherapeutic treatment. ${ }^{13,14}$

Etodolac (ETD) is a non-steroidal anti-inflammatory drug that acts by inhibiting COX-2 enzyme selectively. It has been used to relieve pain and inflammation, associated with rheumatoid arthritis and osteoarthritis. ETD also possesses an antioxidant activity. Tremendous previous studies on ETD have demonstrated its cytotoxic activity on rat tongue carcinogenesis, lymphoblastic leukemia cells, human bladder, and stomach carcinogenesis. ${ }^{15-20}$

It has been reported that ETD as a COX-2 inhibitor when combined with oxaliplatin was found to increase the cell growth inhibition and apoptosis. This combination inhibits the COX-2 enzymatic activity without affecting its level, thus augmenting oxaliplatin therapeutic activity against human colon cancer cells. In another pilot study, ETD serves as an enhancer for the effectiveness of 5fluorouracil. Moreover, ETD was found to inhibit overexpression of both COX-2 and thymidylate synthase, thus inducing a synergistic cytotoxicity with $5 \mathrm{FU}$ on SCC in head and neck. In treatment of human tongue carcinoma, ETD augmented the activity of anticancer drug; carboplatin by significant reduction in fibroblast activation protein-
1 (FAP-1) expression. FAP-1 serves as a blocker for cell death signal, since it is an anti-apoptotic tyrosine phosphatase. $^{12,21}$

Topical drug delivery has been considered as a viable method and one of the attractive approaches for drug delivery in the treatment of skin disorders such as SCs, inflammatory disorders, and other cutaneous infections. It has many privileges such as ease of drug application, localized application at the pathological site of action, reduced side effects, and more relevant in treating skin disorders. Efficient delivery techniques should be elicited to ensure the permeation of therapeutics concentration to the viable epidermal and dermal layers beneath. However, the failure to achieve adequate drug amount at the target skin site restricts the therapeutic benefits of skin drug delivery, despite its potency. ${ }^{22}$

Nanocarriers refer to a huge panel of delivery systems. Nanoparticles might enhance the solubility of hydrophobic loaded active drugs and has shown a superiority in the treatment of many cancers. The promising merit of nanocarrier systems is their capability to exploit the porosity of tumor vasculature and pass directly to tumoral cells. Furthermore, it provides protection of molecules from interactions with other biological components with subsequent improved intratumor drug stability, controlled drug release, and modified pharmacokinetics and biodistribution. Different polymer and lipidbased nanocarriers were evolved, including liposomes, solid lipid nanoparticles, nanostructured lipid carriers (NLCs), nanoemulsions, and polymeric nanoparticles, etc. have been used to encapsulate these impermeable and macromolecular drugs and to enhance their surpassing through the stratum corneum (St.C) barrier. ${ }^{23,24}$

Liposomes are flexible lipid nanovesicles, that can encapsulate both hydrophilic and lipophilic drugs due to their unique structure. Based on this, they enhance the solubility of poorly soluble drugs. Because of their lipid content, they have a marked affinity to keratinocytes, enhancing its penetration into deeper skin layers, thus can act as a penetration enhancer as well as a local depot for sustained drug delivery. ${ }^{3,25}$

NLCs are a class of nanoparticulated drug carriers, composed of a mixture of solid and liquid lipids. The absence of organic solvent and utilization of physiological lipids in their preparation decreases their toxicity. ${ }^{3,23}$

Various polymeric nanoparticles were utilized for topical drug delivery systems. Natural and synthetic polymers were used in nanoparticle' formulations; poly (lactic-co-glycolic acid), chitosan, alginate, and poly( $\varepsilon$-caprolactone), etc. 
Literature survey recorded that alginate nanoparticles (AlgNPs) are not extensively studied as topical drug delivery vehicle. Curcumin-loaded AlgNPs revealed a significant accumulation of curcumin in the upper layers of the skin, asserting its potential for topical delivery of pharmaceutical and cosmetic active agents. ${ }^{2,26}$

Tailored flexible lipid nanovesicles (phosalosomes) based on Phosal ${ }^{\circledR}$ premix as a source of phosphatidylcholine (PC) were prepared. Aqueous core with lipid bilayer coat is perfectly assembled in aqueous medium after dehydrationrehydration processes. Since, surface charge has a crucial rule in drug permeation though skin layers. Both negatively and positively charged phosalosomes (P-Phsoms) were investigated to evaluate the influence of surface charge on skin permeation ability. NLCs were utilized to elucidate the influence of lipid content on drug characteristics.

Various studies reported a controversial between the implementation of lipid vs polymeric nanocarriers for the enhancement of topical drug delivery systems. Since, this is the first study employing ETD in the management of SC, it was encapsulated in either lipid or polymeric nanocarrier in an attempt to achieve the highest therapeutic outcomes.

Accordingly, this study was conducted to elucidate the chemopreventive potential, cytotoxic, and suppression of cellular metastatic activity of ETD-loaded nanocarriers. To achieve such goals, ETD-nanocarriers were developed with enhanced topical delivery potential. ETD was firstly, encapsulated into cationic and anionic lipid vesicles (N-, P-Phsoms), NLCs, and AlgNPs. Full in-vitro characterization was performed. Permeation and localization though human skin layers were assessed and tracked with the aid of confocal laser scanning microscopy (CLSM). In-vitro cytotoxic activity and wound-healing assay were performed on SCC cell line, to provide a proof evidence of the superior anti-tumor activity of the designed nanocarriers. In-vivo chemoprevention efficacy was assessed by measuring the tumor volume, COX-2 m RNA, proliferating cell nuclear antigen (PCNA) mRNA expression, caspase- 3 enzyme activity and acquiring images in an experimental SC mice model.

\section{Materials and methods Materials}

ETD was kindly donated by European Egyptian Pharmaceuticals, Egypt), Phosal ${ }^{\circledR 53}$ MCT (PH) was gifted by lipoid Co. (Ludwigshafen, Germany). Cholesterol (CH), and rhodamine B were purchased from Oxford Lab Chen, India. Tween80, Stearyl amine (SA) were purchased from sigma Aldrich, (Japan). Sodium hyaluronate (HA) (1.5-1.8 million Da) was from Shaanxi Green Bio-Engineering Co., Ltd (China). Formalin 37\% and calcium chloride were purchased from Chem- Lab, (Belgium). Sodium alginate was purchased from Loba Chemie Pvt. Ltd (India). Isopropyl myristate (IPM) was from Fluka (Oslo, Norway). Compritol 888 ATO was provided from Gattefossé (Saint Priest, France). Sterile filtered Dulbecco's modified Eagle's medium (DMEM) high glucose with L-glutamine, and Trypsin EDTA,170,000 U/L were purchased from BioWhittaker (Lonza, Belgium). Fetal bovine serum (FBS), dimethyl sulfoxide (DMSO), and 3-(4,5-dimethylthiazol2yl)2,5-diphenyl tetrazolium bromide (MTT) were purchased from Sigma Aldrich (USA). Ehrlich ascites carcinoma (EAC) parent line was supplied from National Institute of Cancer, Cairo University, Egypt. Alanine aminotransferase (ALT) activity, aspartate aminotransferase (AST) activity, and creatinine assay kits were purchased from Spectrum (Hannover, Germany). Urea assay kit was purchased from Diamond Diagnostics (Hannover, Germany). Caspase-3 colorimetric assay kit was purchased from R\&D systems Inc., Germany. RNeasy Mini Kit (Qiagen, Hilden, Germany). Superscript II reverse transcriptase kit (Invitrogen, USA) and 1X Sybr $^{\circledR}$ Green PCR master mix (Applied Biosystems, USA). Acetonitrile high-performance liquid chromatography (HPLC) grade from Romil Ltd (Cambridge, England). All other chemicals were of analytical grade and used without purification.

\section{Methods}

Preparation of phosalosomes (N-Phsoms, P-Phsoms) Phosalosomes were prepared according to the previously reported thin-film hydration method, with slight modification. $^{27}$ ETD was dissolved in methanol and then added to a chloroform solution of phosal, Tween 80, and $\mathrm{CH}$ (4\% of PC content) in a $500 \mathrm{~mL}$ round-bottomed flask. Rotary evaporator (Rotavapor, type R110, Buchi, Flawil, Switzerland) was used to form thin dry film by evaporation at $40 \pm 5^{\circ} \mathrm{C}$ under reduced pressure. Phosphate-buffer (PB) $\mathrm{pH} 5.5$ was added to rehydrate the produced dried film. Dispersion was then homogenized using high speed homogenizer for $15 \mathrm{mins}$ at $4200 \mathrm{rpm}$ (ULTRA-TURAX T25, IKA Labortechnik, Germany) followed by sonication for 30 mins (Julabo sonicator, model USR3, Julabo Labortechnik, Seelbach, Germany). For the preparation 
of P-Phsoms, SA (10\% of PC) was added to the lipid mixture and the same procedure was followed.

\section{Nanostructure lipid carrier (NLCs)}

NLCs were prepared by the hot melting homogenization method with slight modification. The oil phase (OP), was composed of Compritol ATO (solid lipid), IPM (liquid lipid) in a ratio of $1: 1$ and was heated to $80^{\circ} \mathrm{C}$. The aqueous phase (AP), was composed of Tween 80 and purified water, which was then heated to the same temperature. AP was gently dropped onto the OP under constant agitation $(10,000 \mathrm{rpm}$, for 2 mins) using a thermostated water bath (Stuart, SBS40, Staffordshire, UK), followed by 10 mins sonication. ETD was dissolved in methanol: acetone $(1: 1)$ and added to OP prior to AP addition. $^{23}$

\section{Alginate nanoparticles (AlgNPs)}

AlgNPs preparation was based on the ionotropic gelation of alginate polyanions with calcium chloride following earlier-described method by B. Sarmento et $a{ }^{28}$ and R. Grillo et al, ${ }^{29}$ ETD was added to $117.5 \mathrm{~mL}$ of alginate

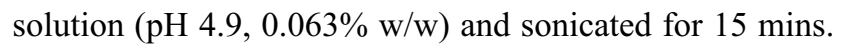
Then, $7.5 \mathrm{~mL}$ of $18 \mathrm{mM} \mathrm{CaCl}_{2}$ was added dropwise to the mixture under magnetic stirring (Ika, Labortechnik, Germany) then formed nanoparticles were incubated for $24 \mathrm{hrs}$. AlgNPS were separated by centrifugation at 14,000 rpm for 30 mins at $4{ }^{\circ} \mathrm{C}$ and redispersed in $\mathrm{PB} \mathrm{pH}$ (5.5).

\section{Physicochemical characterization of nanocarriers} Particle Size (PS), Zeta-Potential (ЦP), Poly Dispersity Index (PDI), and Encapsulation Efficiency EE\%

The Average PS of ETD-nanocarriers and their PDI were analyzed at $25^{\circ} \mathrm{C}$ by dynamic light scattering (DLS) technique using Zetasizer (Malvern Instrument NanoZS, UK). $\zeta \mathrm{P}$ were calculated from the mean electrophoretic mobility obtained using the same instrument in a universal folded capillary cell, with embedded platinum electrodes. All samples were diluted in purified water and measured in thee replicates and results were expressed as the mean value \pm standard deviation (SD). EE\% of ETD-loaded formulations was calculated by with ultrafiltration process, using vivaspin centrifuge filter membrane (4000 rpm for 30 mins) and substitute in the equation. ${ }^{24}$

$$
\mathrm{EE} \%=\frac{\begin{array}{c}
\text { the total drug content }- \text { amount of } \\
\text { drug in the filtrate }
\end{array}}{\text { the total drug content }} \times 100 \%
$$

HPLC qualification method: A simple sensitive isocratic quantitative and stability indicating HPLC method for ETD. The HPLC instrument (1200 series Agilent Technologies, California, USA) equipped with a photodiode array detector. The column used was a reverse phase $\mathrm{C} 18$ column (Agilent 5HC-C18 (2) $250 \times 4.6 \mathrm{~mm}$ ). The mobile phase consisted of acetonitrile: Phosphate- buffer saline (PBS) (4 M, pH7.4) in a ratio of $(35: 65)$ at a flow rate $1 \mathrm{~mL} / \mathrm{min}$. Analysis was carried out at a wavelength of $278 \mathrm{~nm}$. Standard solutions of ETD over the range 3-30 $\mu \mathrm{g} / \mathrm{mL}$ were prepared by subsequent dilution of stock standard solution of ETD in PBS (pH7.4) with a final concentration of $30 \mu \mathrm{g} / \mathrm{mL}$. A calibration curve (peak area vs drug concentration) was constructed by running the working standard solutions in the mobile phase, $\left(\mathrm{R}^{2}=0.9978\right) .{ }^{30}$

\section{Morphological assessment and thermal analysis}

Transmission and scanning electron microscopic (TEM and SEM) imaging of ETD-loaded nanocarriers and differential scanning calorimeter (DSC) analysis were performed as stated in Supplementary material.

\section{In-vitro release study}

In-vitro release profiles of ETD from the different nanocarrier dispersions were studied using semi-permeable dialysis membrane with a molecular weight cut off 12,000-14,000 Da (Serva, Heidelberg, Germany) crafted as bags. Known amount of each of the prepared formulations equivalent to $10 \mathrm{mg}$ ETD was filled in the dialysis bag and suspended in beaker containing $100 \mathrm{~mL}$ PBS $(\mathrm{pH} 7.4)$ as a release medium, placed in a shaking thermo-stated water bath at $32^{\circ} \mathrm{C}$ and shaken at $75 \mathrm{rpm}$. At predetermined intervals and after $24 \mathrm{hrs}$, samples of $2 \mathrm{~mL}$ each were withdrawn and compensated with the same volume of preheated fresh dissolution medium. The total amount of ETD released was analyzed by HPLC. Additionally, the remained formulations in the dialysis bag were analyzed to determine unreleased. The results were expressed as mean \pm S.D. Data were presented as $\%$ ETD release against time in $\mathrm{h}$. In addition, time for $50 \%$ drug to be released $\left(\mathrm{T}_{50}\right)$ is used as a tool for comparison.

\section{Ex-vivo permeation and retention study through human skin \\ Preparation of human skin samples}

All protocols and procedures for acquiring and processing the human skin were carried out in accordance with the ethical guidelines of Alexandria University Hospital, 
Alexandria, Egypt. Written Informed consent was taken from the patient and all drugs used in the research are approved by the Egyptian ministry of health. Full thickness human skin was obtained from a female patient undergoing abdomen reduction plastic surgery. After trimming the subcutaneous fatty tissue, the skin was then washed with Ringers' solution, dried, wrapped in an aluminum foil and stored at $-20^{\circ} \mathrm{C}$ until use. Prior to the experiment, skin specimens were washed with PBS $(\mathrm{pH7} .4)$ and allowed to come to room temperature (RT).

\section{Permeation study}

Skin specimens were sandwiched between two champers of a modified vertical Franz diffusion assembly with an effective diffusional area of $3.14 \mathrm{~cm}^{2}$ and with St.C side upwards. The receptor chamber was filled with $8 \mathrm{~mL}$ PBS (pH7.4) avoiding air bubbles. The whole assembly was kept in thermostatically shaking water bath at $32 \pm 0.5^{\circ} \mathrm{C}$ and $75 \mathrm{rpm}$. After equilibration for $30 \mathrm{mins}$, an amount equivalent to $1 \mathrm{mg}$ ETD of each of the investigated nanoformulations was placed over the skin in the donor champers without application of any occlusive conditions. The whole release media were sucked with a syringe from receiver chamber's side tube at appropriate time interval (1-8, $24 \mathrm{hrs})$ and replaced with fresh medium. At each time interval, fresh medium of $\mathrm{pH} 7.4$ was immediately refilled to maintain constant volume. Collected samples were then filtered through polytetrafluoroethylene 0.22 $\mu \mathrm{m}$ syringe filters and quantified by HPLC. Each study was performed in triplicates. Data as cumulative amount of ETD permeated in $\mu \mathrm{g} / \mathrm{cm}^{2} / \mathrm{h}, J_{\mathrm{ss}}$ (the drug permeation flux at steady state), the apparent permeability coefficient $(\mathrm{Kp})$, and the diffusion coefficient " $\mathrm{D}$ " were calculated according to Marwa A. et al. ${ }^{31}$

\section{Skin retention study}

At the end of the permeation study, skin deposition study was performed where amount of ETD retained within the skin layers was restored and quantified. Skin specimens were removed from the Franz cell, cleaned with gauze gently, and then rinsed with PBS (pH7.4) to remove any residual formulation. St.C was then removed gently by tape stripping using five strips for eight times (Transpore ${ }^{\circledR}$ tape $3 \mathrm{M}$ company, St. Paul, MN), while epidermis and dermis were kept together. Skin layers were then immersed in methanol for $24 \mathrm{hrs}$, and then sonicated for $1 \mathrm{hr}$ at RT. Extracts were then filtered through a $0.22 \mu \mathrm{m}$ syringe filter and analyzed for ETD retained $\left(\mu \mathrm{g} / \mathrm{cm}^{2}\right) .{ }^{32}$

\section{Tracking ex-vivo skin penetration pathway and localization using CLSM}

The CLSM instrument was utilized to track fluorescence marker pathway, its distribution in skin layer, inter or intracell localization. Rhodamine B (RH) was used as a fluorescent dye and was loaded into P-Phsoms, N-Phsoms, NLCs, and AlgNPs. Permeation though human skin was set up using the Franz diffusion assembly as previously described (section Ex-vivo permeation study on human skin). Five hundred $\mu \mathrm{L}$ RH-labeled formulation was applied to the surface of the skin and the whole assembly was wrapped with aluminum foil to avoid light. After 6 hrs, the skin specimens were removed washed gently with buffer, dried gently with cotton swap to remove excess dye, stained skin specimens were cut and fixed in $10 \% \mathrm{v} / \mathrm{v}$ formalin solution. Afterward, paraffin wax was used to cover skin specimens forming cubes. By the aide of microtome, skin specimens' cubes were sliced into a number of sections each $6 \mu \mathrm{m}$ thick. Sections were stained with $1 \mathrm{mg} / \mathrm{mL}$ of 4,6-diamidino-2-phenylindole (DAPI) for 10 mins at RT. After washing with saline, the cross-sections of the skin samples were imaged by CLSM (Leica DMi8, Leica CMs$\mathrm{GmbH}$, Germany) with dual excitation band of DAPI (410$480 \mathrm{~nm})$ and RH (530-615 nm) under a 63× objective lens. The fluorescence intensity of each image was calculated using equipped Leica software.

\section{Assessment of skin irritation potential of the formulations}

Herein, to investigate the local safety of different ETD-loaded nanocarriers, skin histological assessment was carried out using aforementioned modified Franz diffusion cell method (section Ex-vivo permeation study on human skin). At the end, skin specimens were prepared and sliced as mentioned under the previous section. Prepared slices were stained with hematoxylin and eosin (H\&E) and examined using a light optical microscope equipped with a digital camera for histological changes detection (Olympus EX-41, equipped with Olympus DP20 camera, USA). ${ }^{33}$

\section{In-vitro anticancer activity}

ETD solution and ETD-loaded formulations were assessed for their potential cytotoxic activity against squamous cell carcinoma cells (SCC 9) using the MTT assay.

SCC cells were purchased from the American Type Culture Collection (ATCC), were cultured in $75 \mathrm{~cm}^{2}$ flasks in DMEM high glucose with L-glutamine medium, 
supplemented with 10\% FBS, and trypsin EDTA and maintained at $37^{\circ} \mathrm{C}$ under $5 \% \mathrm{CO}_{2}$, and $95 \%$ humidity for $24 \mathrm{hrs}$.

For MTT assay, $200 \mu \mathrm{L}$ cell dispersion containing 7000 cells were seeded in each well of 96-well plate. Cells were grown till reaching 80-90\% confluency, after $24 \mathrm{hrs}$. Then, they were treated by serial concentrations of the tested formulations and left for another $24 \mathrm{hrs}$. Then $0.01 \mathrm{~mL}$ MTT solution was added to the wells and incubated for another 4 hrs at $37^{\circ} \mathrm{C}$. The culture medium was removed and DMSO was added to dissolve formed crystals. The absorbance was measured at $490 \mathrm{~nm}$ on Bio Rad Microplate Reader (Benchmark, USA). The percentage cell viability at each concentration was calculated using linear regression analysis. Accordingly, half maximal inhibitory concentration $\left(\mathrm{IC}_{50}\right)$ value of each formula (The dose of the formulation that inhibited $50 \%$ cell viability) was determined. ${ }^{34,35}$

\section{Wound-healing assay (Scratch assay)}

In-vitro cell migration assay was conducted to figure out the ability of ETD to inhibit cancer cell migration. For this purpose, SCC cells were seeded in 24-well plate and cultured in complete medium at $37^{\circ} \mathrm{C}$ for $24 \mathrm{hrs}$ till the formation about $100 \%$ confluent monolayer. Wound was created by scratching using yellow pipette tip as a cell-free zone and washed twice with saline. The cells were furtherly incubated at $37^{\circ} \mathrm{C}$ with each of free ETD solution in sterile water and ETD-loaded nanoformulation (ETD-N-Phsoms) dispersion $(30 \mu \mathrm{g} / \mathrm{mL})$ and compared with untreated cells. Cell migration was then examined after $24 \mathrm{hrs}$ by phase contrast imaging with an inverted microscope (Zeiss, Axiovert 25, Germany). The \% cell migration or wound-healing inhibition represents the difference between width of the wound at $24 \mathrm{hrs}$ and the original wound width relative to the original width at $0 \mathrm{hrs}^{36}$

\section{In-vivo chemoprotective potential}

\section{Animals}

A total of 28 mature female albino mice (average 20-25 g) were enrolled in the in-vivo anti-tumor activity and toxicity studies. The animals were kept in standard metal cages at $21 \pm 1^{\circ} \mathrm{C}$, relative humidity $(65 \%)$ with a $10 \mathrm{hrs}$ light $/ 14 \mathrm{hrs}$ dark cycle. Before the experiment, animals were allowed to accommodate to their environment for 2 weeks. The animals were kept at the animal house of the Faculty of Medicine, Alexandria, Egypt, and were given standard chow and water ad libitum for the study period. Approval of ethical committee was obtained, and animals were handled according to the ethical guidelines of Alexandria University (Au:06/2019/2/12/2/39). Furthermore, animal experiments were in compliance with the ARRIVE guidelines and in accordance with the UK Animals (Scientific Procedures) Act, 1986. and associated guidelines, EU Directive 2010/63/EU for animal experiments.

\section{Experimental design}

The Ehrlich's ascites carcinoma (EAC) cell line was purchased from the Tumor Biology Department, National Cancer Institute, Cairo University (Cairo, Egypt). EAC cells were handled under aseptic conditions and their viability was confirmed using Trypan blue dye exclusion technique. ${ }^{37}$ For induction of intradermal solid tumor, EAC cells were suspended in saline which contained 2.5 million cells per 0.1 $\mathrm{mL}$ of the suspension. At the day of the experiment, mice were randomly divided into four groups, seven mice each. The groups encompassed: Group I; the negative control group (healthy mice that received no topical treatment), and Groups II, III, and IV that received topical treatment. Group II; the positive control group, received topical saline treatment while Groups III and IV were treated topically once daily with free ETD solution in acetone (free ETD, $25 \mathrm{mg} / \mathrm{mL}$ ), and ETD-Phsoms loaded HA gel as ETD nanoformulation (ETD nano, $10 \mathrm{mg} / \mathrm{mL}$ ), respectively. After 3 weeks of topical treatment, Groups II, III, and IV were inoculated intradermally with $0.1 \mathrm{~mL}$ of the EAC suspension bilaterally on the upper dorsal side and treatment was continued during tumor formation ( 8 days). In the meantime, the negative control group received intradermal injection of saline instead. At day 8 after inoculation with the EAC cells, tumor growth was confirmed, and the experiment was terminated. ${ }^{38}$

\section{Collection of serum samples and dissection of tumor discs}

At the end of the experiment (day 28), blood samples were withdrawn from each mouse from the orbital sinus under light anesthesia. Blood samples were allowed to clot and were centrifuged at $3000 \mathrm{rpm}$ for $15 \mathrm{mins}$. The obtained serum samples were stored at $-20^{\circ} \mathrm{C}$ for determination of liver functions (ALT and AST) and kidney functions (urea and creatinine). Mice were then sacrificed by cervical dislocation and tumor discs were dissected, rinsed in ice-cold saline and weighed. Relative tumor weight (\%) was calculated as the ratio of the tumor weight to the body weight. For each animal, one tumor disc was stored at $-80^{\circ} \mathrm{C}$ for determination of the mRNA expression of COX-2, the molecular target for ETD, and PCNA, proliferation marker, and for the assessment of caspase 3 activity (a marker of apoptosis). The 
other tumor sample was fixed in $10 \%$ phosphate-buffered formalin for histopathological examination.

\section{In-vivo toxicity study}

Being an important index to the overall health state, body weight of each animal was recorded at the beginning of the study and continued weekly afterward using an electronic balance (Precisa Instrument, Dietikon, Switzerland). Additionally, animals were observed for any clinical signs of toxicity (i.e. skin, fur, or mucous membrane changes, diarrhea, neuronal, cardiac, or others) and mortalities following the administration of the formulation and though the study period. After animals' sacrifice, major organs (liver, spleen, kidneys, brain, etc.) of control and treated animals were examined for any significant changes in shape and texture. Furthermore, relative organs' weight was calculated as the ratio of the organ weight to the body weight. Organ functions were determined biochemically as well as mentioned above.

\section{Biochemical tests}

Serum ALT, AST, Serum creatinine activities were measured spectrophotometrically using Spectrum Assay Kit, while serum urea level was determined by enzymatic colorimetric method using Diamond Diagnostics Assay Kit. Tumor COX-2 and PCNA mRNA expression was assessed using quantitative reverse-transcription PCR with glyceraldehyde 3 phosphate dehydroenase (GAPDH) as the housekeeping gene. As a marker for apoptosis, caspase-3 enzymatic activity was measured by colorimetric reaction using R\&D Systems kit. The specific primer pairs for COX-2, PCNA, and GAPDH are listed in Table 1. Detailed biochemical tests were given in the Supplementary material.

\section{Histopathological and immunohistochemistry (IHC) examination}

Excised tumors were processed into H\&E histological examination for the assessment of tumor tissue as well as ETD treatment effect as previously described under section (Assessment of skin irritation potential). IHC was done on positive control group tumors using the avidin-biotin-peroxidase complex methodology to figure out the cancer type. Each slide was immersed into absolute ethanol for 15 mins. Slides were rehydrated in a series of graded ethanol. Tissue sections were stained with the primary antibodies: MultiCytokeratin AE1/AE3 (Ready to use primary antibody, mouse anti-human, monoclonal antibody, P0012) and HMB-45 (Ready to use primary antibody, mouse antihuman, monoclonal antibody, P0027) use the Bond-Max
Table I The specific primer pairs for COX-2, PCNA, and GAPDH with accession number

\begin{tabular}{|l|l|l|}
\hline Target & Sequence & $\begin{array}{l}\text { Accession } \\
\text { number }\end{array}$ \\
\hline COX-2 & $\begin{array}{l}\text { CCACTTCAAGGGAGTCTGGA } \\
\text { (forward) } \\
\text { CCACTTCAAGGGAGTCTGGA } \\
\text { (reverse) }\end{array}$ & NM_0III98.3 \\
\hline PCNA & $\begin{array}{l}\text { AGATGCCGTCGGGTGAAT } \\
\text { (forward) } \\
\text { TCTCTATGGTTACCGCCTCCT } \\
\text { (reverse) }\end{array}$ & NC_000068 \\
\hline GAPDH & $\begin{array}{l}\text { AGCTTGTCATCAACGGGAAG } \\
\text { (forward) } \\
\text { TTTGATGTTAGTGGGGTCTCG } \\
\text { (reverse) }\end{array}$ & NC_000072 \\
\hline
\end{tabular}

Abbreviations: COX-2, cyclooxygenase-2; PCNA, proliferating cell nuclear antigen; $\mathrm{GAPDH}$, glyceraldehyde 3 phosphate dehydrogenase.

fully automated immunostainer, Leica Biosystems, USA. Finally, the sections were counterstained by Hematoxylin. In the IHC run, the skin epidermis was used as the internal positive control for Multi-Cytokeratin AE1/AE3 antibody and a case of malignant melanoma was used as the positive control for HMB-45 antibody. Negative control with omitted primary antibody was also included in the IHC run. ${ }^{39}$

\section{Data analysis}

In-vivo data were analyzed using IBM SPSS software package Version 20 (IBM Corporation, Armonk, NY, USA). Comparison between the studied groups was carried out using F-test (ANOVA) and post hoc test (Tukey). Difference was considered statistically significant at a level of $P$-value $<0.05$.

\section{Statistical analysis}

Results were expressed as the mean of three independent experiments \pm SD. Data analysis was carried out using one-way analysis of variance test (ANOVA) followed by two-tailed test to assess the level of significance among each two tested formulations using Microsoft Office Excel 2016. Difference was considered statistically significant at a level of $P$-value $<0.05$.

\section{Results and discussion Preparation of different nanocarriers}

Since sufficient high solubility of drug in lipid matrix is a prerequisite for good drug loading as well as for suitable association with the lipid. It has been reported 
that ETD displayed higher solubility in IPM over other liquid lipids and in Tween 80 as a preferential surfactant. Preliminary solubility study of ETD in Phosal indicated higher solubility of $188.03 \pm 8.84 \mathrm{mg} / \mathrm{mL}^{16}$

ETD is a poorly water-soluble drug with a pka 4.76. Being a hydrophobic drug, could be considered as a good candidate to be incorporated in the lipid bilayer of lipid vesicles. Therefore, two different lipid nanovesicles; namely (NPhsoms and P-Phsoms), and NLCs were prepared to study the influence of surface charge of Phsalosomes and lipid content of NLCs on drug loading and performance. In addition, polymeric nanoparticles (AlgNPs) were prepared to implement the difference between lipid and polymer on drug delivery. Since minimum research studies have employed alginate polymer for skin delivery and in order to ensure the reproducibility of AlgNPs characteristics and yield, formulation conditions were optimized and kept constant. The optimal conditions for maximum AlgNPs production were $\mathrm{pH} 4.9,18$ $\mathrm{mM}$ concentration of $\mathrm{CaCl}_{2}$, and $24 \mathrm{hrs}$ incubation time.

\section{PS, PDI, לY, and EE\%}

All nanoformulations showed a sufficiently good polydispersity (PDI 0.3) (Table 2), indicating a moderately polydisperse systems which confirmed with transmission electron microscope (TEM) photographs (Figure 1A). The higher PDI of NLCs may be related to the presence of IPM, which offered a substantial solubility to ETD, resulting in higher PDI value. $^{40,41}$

The mean diameter of the various nanocarriers dispersions ranged from 202 to $267 \mathrm{~nm}^{24}$ Particle sizes of

Table 2 The average diameter of the formulations $(\mathrm{nm})$, PDI value, zeta-potential $(\mathrm{mV})$, percentage entrapment efficiency (EE\%)

\begin{tabular}{|l|l|l|l|l|}
\hline Formulation & Average diameter $(\mathbf{n m})$ & Zeta-potential $\mathbf{( m V})$ & PDI & EE\% \\
\hline N-Phsoms & $202.96 \pm 2.37$ & $-24.8 \pm 4.16$ & $0.321 \pm 0.015$ & $88.52 \pm 0.07$ \\
P-Phsoms & $260.15 \pm 26.23$ & $35.4 \pm 4.73$ & $0.339 \pm 0.04$ & $68.95 \pm 0.68$ \\
NLCs & $267.43 \pm 5.17$ & $-11.8 \pm 3.07$ & $0.360 \pm 0.017$ & $88.86 \pm 0.56$ \\
AlgNPs & $248.06 \pm 23.73$ & $-14.3 \pm 7.64$ & $0.353 \pm 0.02$ & $98.63 \pm 1.97$ \\
\hline
\end{tabular}

Abbreviations: PDI, Poly Dispersity Index; NLCs, nanostructured lipid carriers; AlgNPs, alginate nanoparticles.

A
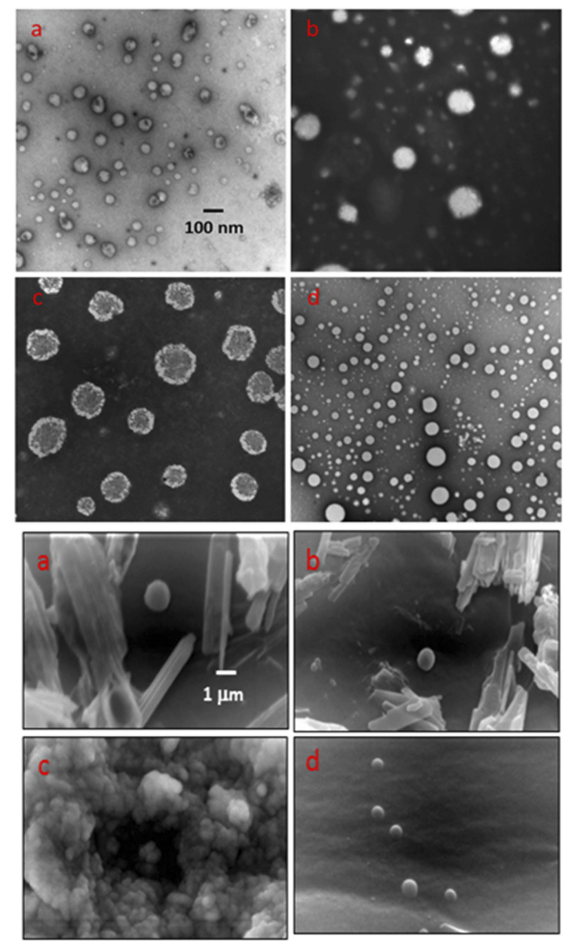

B

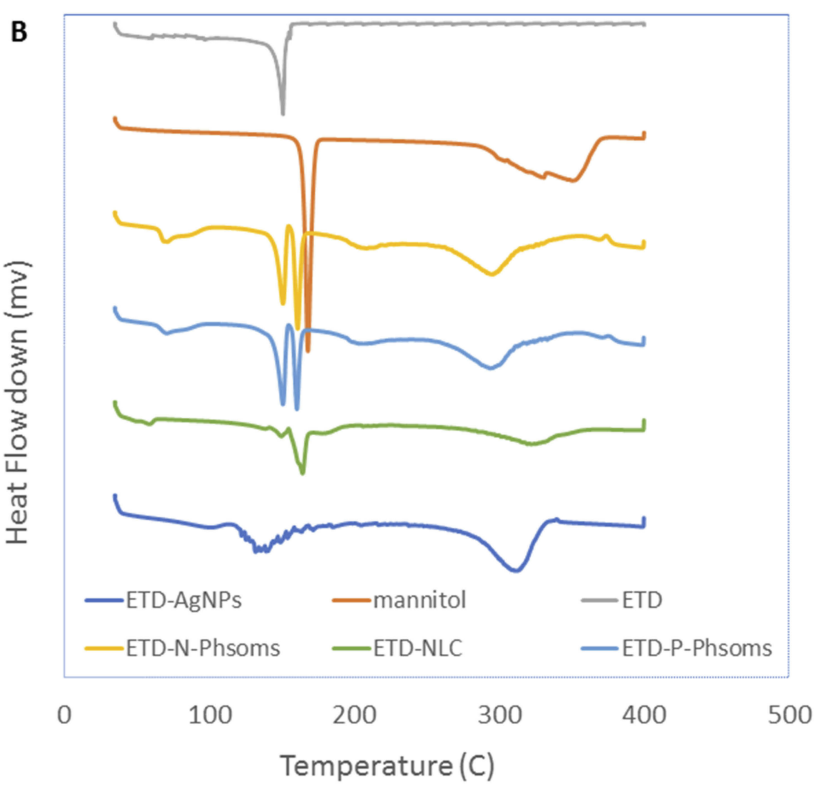

Figure I (A) TEM and SEM images of different ETD nanoformulations; (a) N-Phsoms, (b) P-Phsoms, (c) NLCs, and (d) AlgNPs. TEM images was at 7500× and scale bar is $100 \mathrm{~nm}$. While SEM images were at 7500x magnification power and scale bar is I $\mu \mathrm{m}$. (B) DSC thermograms of Mannitol, ETD, N-Phsoms, P-Phsoms, NLCs, and AlgNPs. Abbreviations: ETD, Etodolac; NLC, nanostructured lipidcarriers; TEM, transmission electron microscope; SEM, scanning electron microscope; AlgNPs, alginate nanoparticles; DSC, differential scanning calorimeter. 
nanocarriers upon DLS measurement were depicted in Table 2. Based on the literature, formulations intended for dermatological use should exhibit sufficiently small PS to penetrate efficiently through the skin layers, which is assisted by their large surface area and high mechanical strength. ${ }^{26}$

N-Phsoms showed a smaller PS compared to P-Phsoms, reflecting the impact of SA addition. This agrees with a previously reported study, where positive phytosphingosine nanoemulsions had larger particle size than negatively charged Tween 80 nanoemulsions. ${ }^{42}$ NLCs demonstrated a smaller diameter $(267.43 \pm 5.17)$ than the reported value $(285.3 \pm 28.9 \mathrm{~nm})$, which could be referred to the increased amount of liquid lipid in the formulation; leading to a higher system emulsification and reduced size. AlgNPs showed particle size very close to the reported values. $^{43}$ Regarding $\zeta \mathrm{P}$ which is a surface charge parameter that represents the electrostatic mobility of the particles. It may depend on many factors; nature of particle, the external medium composition, arrangement of lipid molecules on the surface of nanoparticles, interaction with surfactant as well as adsorption of surfactant molecules on interfacial surface. In addition, researchers have reported that unentrapped oil droplets may accumulate at the surface of particles and may contribute to zeta potential. $^{40,44} \mathrm{~N}-\mathrm{Phsoms}$, NLCs, and AlgNPs imparted a negative $\zeta \mathrm{P}(-11.8$ to $-24 \mathrm{mV})$. Surface modification of N-Phsoms with SA produced P-Phsoms with a high positive $\zeta \mathrm{P}(35.4 \pm 4.73 \mathrm{mV})$. Nevertheless, some authors proved that a $\zeta \mathrm{P}$-value of $-10 \mathrm{mV}$ could be sufficient for nanosystems stability. Herein, ¿P-values confirmed the physical stability of the prepared nanovesicles. ${ }^{26}$

Encapsulation of ETD in nanocarriers could be such an interesting approach to increase its skin localization potential. As recorded in Table 2, all nanocarriers revealed good EE\%. N-Phsoms exhibited no significant $(P>0.05)$ difference in EE\% compared to NLCs. AlgNPs reached up to $98 \%$, which is in agreement with EE\% of hydrophobic curcumin in the same polymeric nanoparticle $(95 \%){ }^{26}$

\section{Morphological assessment using TEM and SEM}

TEM and SEM photographs demonstrated single, spherically shaped unilamellar vesicles. The bright color with a little folded structure in TEM images demonstrates the hollow core of the vesicles (Figure 1A). NLCs as almost spherical with dark colored core. The dark core may be related to drug solubilized in lipid phase. ${ }^{40}$
TEM size measurements indicate an average diameter of $79.66 \pm 19.13 \mathrm{~nm}$ and $146.83 \pm 18.51 \mathrm{~nm}$ for N-Phsoms and P-Phsoms, respectively. NLCs and AlgNPs recorded a diameter of $194.83 \pm 18.89 \mathrm{~nm}$ and $103.33 \pm 20.07 \mathrm{~nm}$, respectively. Since DLS measures the hydrodynamic diameter of the particles, its measurements were higher than measures obtained from TEM images, which could be referred to the coating effect that decreased the particle diffusivity, thus resulting in a larger hydrodynamic size. In addition to the influence of drying process exhibited during TEM test leading to size shrinkage. ${ }^{45,46}$ Accordingly, it can be concluded that all prepared ETD-loaded nanocarriers possess uniform nanometric size which could be maintained after lyophilization.

\section{Thermal analysis using DSC}

DSC thermograms obtained by ETD, mannitol, and freezedried formulated nanoparticles are shown in Figure 1B. ETD thermogram showed a sharp endothermic peak at $149.49^{\circ} \mathrm{C}$, corresponding to the melting point of ETD and reflecting its crystalline nature. In thermograms of both N- and P-Phsoms, ETD endothermic peak with lesser intensity was presented, revealing the persistence of its crystalline form with no interaction with other ingredients. Absence of endothermic peak of ETD in the NLCs and AlgNPs formulations confirms the conversion of ETD from its crystalline state to its molecularly dispersed amorphous state within the nanoparticle formulations. ${ }^{47}$

\section{In-vitro release study}

One of the goals of the in-vitro release study was to evaluate the efficiency of the formulated nanocarriers in encapsulating ETD and preventing its robust leakage from the core to the surrounding medium. Figure $2 \mathrm{~A}$ shows the $\%$ cumulative release of ETD from formulated nanocarriers over $24 \mathrm{hrs}$. At $8 \mathrm{hrs}$, both N- and P-Phsoms showed less than $80 \%$ of ETD released to the receiver medium $\left(\mathrm{T}_{50}=4.61, \mathrm{~T}_{50}=7.33 \mathrm{hrs}\right.$, respectively), which is in accordance with the literature. ${ }^{48}$ NLCs showed a quick release $\left(\mathrm{T}_{50}=2 \mathrm{hrs}\right)$ of ETD as reported NLCs may display the highest burst drug release as a sequence of surfactant and the related decreased surface tension and thereby NLCs instability or may be attributed to the localization of ETD within the surfactant polar interface rather than the lipid matrix. ${ }^{43,49}$ ETD release from AlgNPs was rapid during the first $3 \mathrm{hrs}\left(\mathrm{T}_{50}=2.10 \mathrm{hrs}\right)$ and then gradually decreased after 3 hrs. This can be explained as at initial phase, ETD rapidly diffused though the swollen alginate matrix. Then 

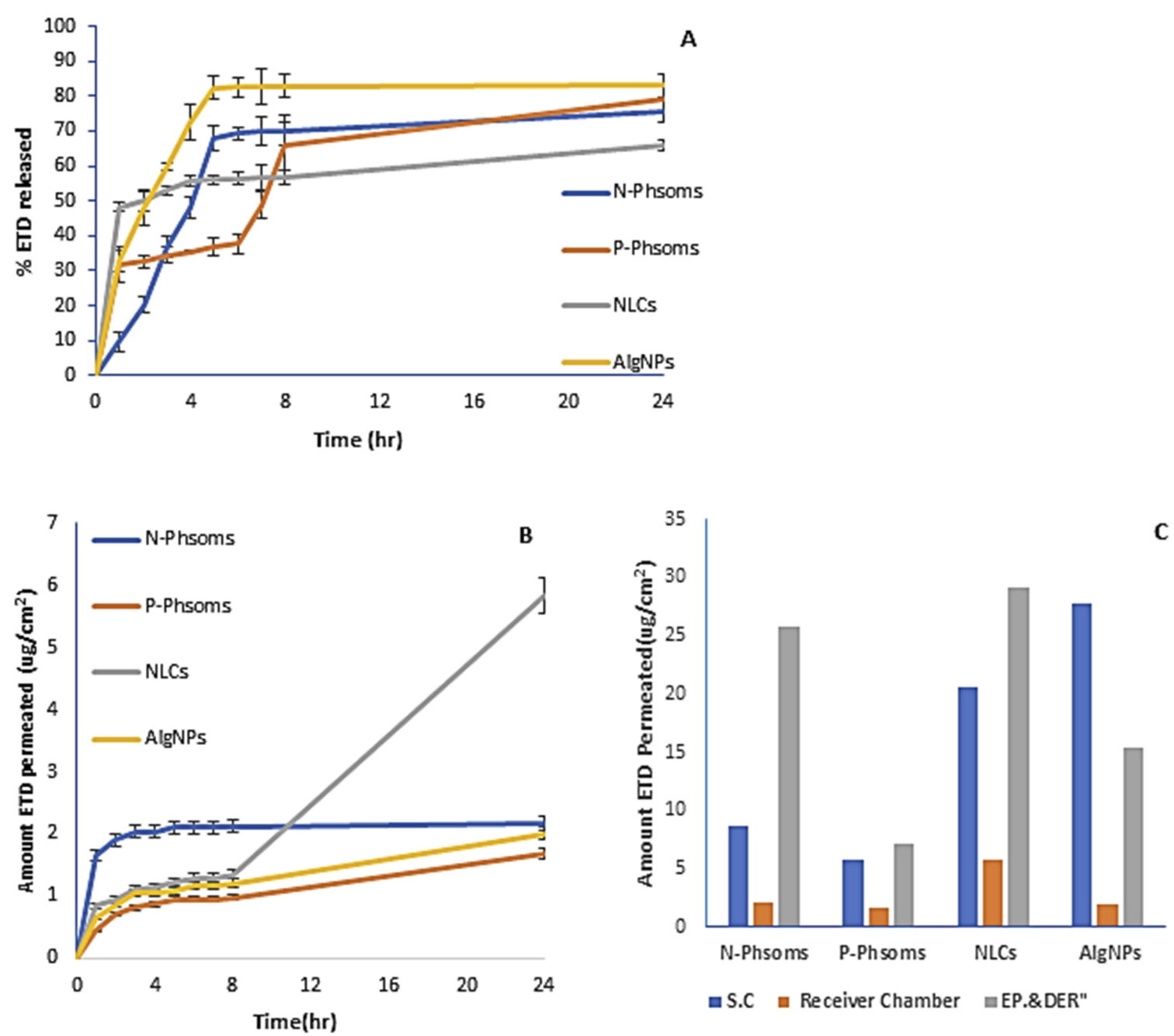

Figure 2 (A) In-vitro release profiles of N-Phsoms, P-Phsoms, NLCs, and AlgNPs in PBS (pH 7.4) using Franz diffusion technique for 24 hrs at $32^{\circ} \mathrm{C}$ and 75 rpm. (B) Ex-vivo cumulative amount $\left(\mu \mathrm{g} / \mathrm{cm}^{2}\right)$-time profiles of ETD permeated from N-Phsoms, P-Phsoms, NLCs, and AlgNPs systems across human abdominal skin using Franz diffusion test over $24 \mathrm{hrs}$. (C) Amount of ETD retained $\left(\mu \mathrm{g} / \mathrm{cm}^{2}\right)$ in St.C, Epidermis and dermis, and in receiver chamber after 24 hrs of experiment. Each value represents the mean \pm SD ( $\mathrm{n}=3$ ).

Abbreviations: NLCs, nanostructured lipid carriers; AlgNPs, alginate nanoparticles; PBS, phosphate buffer saline; ETD, etodolac; St.c, stratum corneum.

subsequently, a sustained release was obtained. The same release profile was obtained as silk sericin was formulated in AlgNPs and the maximum amount released was increased by increasing silk sericin loading. All formulations showed a biphasic release features, that would be appropriate for the prolonged therapeutic influence though transdermal application. The initial drug burst release may be attributed to poorly entrapped drug, or drug adsorbed onto the outside of the particles. ${ }^{50,51}$

\section{Ex-vivo permeation and retention study though human skin}

The potential ability of the elaborated nanocarriers in enforcing ETD skin penetration and retention across human skin layers was investigated. The various nanoformulations of
ETD can be compared regarding the depth and extent of the penetration though the various human skin layers.

\section{Permeation study}

All fabricated formulations displayed minimum amount of ETD permeated (Figure 2B) over $24 \mathrm{hrs}$. The permeation parameters results revealed that permeation flux (Jss) and permeability coefficient (Kp) from NLCs $\left(22.41 \mu \mathrm{g} / \mathrm{cm}^{2} / \mathrm{h}\right.$, $44.88 \mathrm{~cm}^{2} / \mathrm{h}$ ) were the highest compared to the other nanoformulations (Table 3). Higher amount of ETD was permeated after $24 \mathrm{hrs}$ (Figure 2C) from NLCs with about 2.67 folds increase than N-Phsoms. All other formulations showed insignificant increase in cumulative ETD permeated after $24 \mathrm{hrs}(P>0.05)$. These results may be explained as the intimate interaction between NLCs and St.C may form an adhesive layer that may occlude skin surface, 
Table 3 Permeation kinetic parameters of N-Phosoms, P-Phsoms, NLCs, and AlgNPs through abdominal human skin

\begin{tabular}{|l|l|l|l|}
\hline Formulation & $\begin{array}{l}\mathbf{J}_{\mathrm{ss}} * 10^{-2} \\
\left(\boldsymbol{\mu g} / \mathbf{c m}^{\mathbf{2}} / \mathbf{h}\right)\end{array}$ & $\begin{array}{l}\mathrm{Kp} * 10^{-6} \\
(\mathbf{c m} / \mathbf{h})\end{array}$ & $\begin{array}{l}\mathbf{D} * 10^{-5} \\
\left(\mathbf{c m}^{2} / \mathbf{h}\right)\end{array}$ \\
\hline N-Phsoms & 4.11 & 8.22 & 2.58 \\
P-Phsoms & 5.47 & 10.94 & 3.43 \\
NLCs & 22.41 & 44.88 & 14.07 \\
AlgNPs & 6.33 & 12.66 & 3.97 \\
\hline
\end{tabular}

Abbreviations: NLCs, nanostructured lipid carriers; AlgNPs, alginate nanoparticles.

increase its hydration, resulting in reduced corneocyte, and finally, the loose structure greatly enhances drug penetration into deeper skin layers. In addition to the skin penetration enhancing effect of IPM used in its formulation. ${ }^{41,52}$

Skin retention into the St.C, epidermis, and dermis Loaded nanovesicles retained in St.C were in order of AlgNPs $>$ NLCs $>$ N-Phsoms $>$ P-Phsoms. On the other hand, the order in epidermis and dermis deposition was NLCs $>$ N-Phsoms $>$ AlgNPs $>$ P-Phsoms (Figure 2C). The higher ETD-AlgNPs accumulation in St.C (27.73 \pm 2.72 ) could be referred to their rigid structure, causing them to be retained in the upper layer. On the contrary, flexible lipid nanoparticles were able to penetrate deep into intact skin. This may be related to the presence of biocompatible phospholipids in their composition and their vesicular nature. ${ }^{53,54}$

N-Phsoms showed 1.5 and 3.6 folds increase in the amount of ETD deposited in St.C, epidermis, and dermis, respectively compared to P-Phsoms. For P-Phsoms, despite the presence of phospholipid and their vesicular structure, their low retention and permeation values may be attributed to their positive charge. Authors were agreed on the impact of the particles' surface charge on their skin penetration. Some suggested that skin penetration of negatively charged nanoparticles is more difficult than positively charged ones as positively charged nanoparticles expected to electrostatically bind to negatively charged skin, increasing their skin penetration. While, others concluded that negatively charged liposomes increase the betamethasone penetration into epidermis and dermis 2.7 times positively charged liposomes as the repulsive forces created between the nanocarriers and the skin, create channels through which drug can permeate. ${ }^{42,55}$ Noteworthy, several factors; such as lipid composition, particle size, and surface charge were proved to affect nanocarriers penetration and deposition into skin layers. Lipid vesicles adapt different routes to penetrate the skin. They could penetrate though follicular pathway or might break at the SC surface enabling phospholipids penetration within it, thus enhancing permeation of the drug load. Moreover, vesicles might adapt "adsorption/fusion" pathway, where they adsorbed to the skin surface; permitting active molecule to diffuse through the skin layers. ${ }^{56,57}$

\section{Tacking ex-vivo skin penetration pathway and localization using CLSM}

CLSM was utilized to observe the distribution of RH-loaded nanovesicles in the skin layers, to get information about the extent of skin penetration and possible pathway as CLSM Permits skin structure to be studied in three dimensions with very high accuracy. ${ }^{58}$

Microscopic imaging of the cross-section skin slices is in a perpendicular direction to the skin allowed us to reveal the distribution pattern of RH-loaded nanovesicles in the subsequent layers of the skin including the St.C, epidermis, dermis, and deep dermis (Figure 3A). RH-labeled nanocarriers exhibited red fluorescence, while viable skin layers 'cells exhibited blue fluorescence. Comparing N-Phsoms and P-Phsoms (Figure 3A (a, b)), N-Phsoms facilitated the diffusion of RH though St.C and viable epidermis and dermis and uniformly distributed within them with a significant amount in St.C (88.75 $\left.\pm 1.58 \mathrm{Pixel} / \mu^{2}\right)(P<0.005)$ (Figure $\left.3 \mathrm{~B}\right)$. In addition, more N-Phsoms were diffused to deeper skin layers, about $26.86 \pm 3.77 \mathrm{Pixel} / \mu^{2}$ at $200-300 \mu \mathrm{m}$ depth. On contrary, upon using P-Phsoms only small amount was imaged at lower portion of the upper viable layers $(0$ $100 \mu \mathrm{m}) 11.61 \pm 7.54 \mathrm{Pixel} / \mu \mathrm{m}^{2}$ (Figure 3B). This is with agreement with a previous study, when negatively charged liposomal formulation showed a significant accumulation of the fluorescence intensity in the deeper dermal layers. ${ }^{24}$ Most RH-loaded N-Phsoms could penetrate deeply though the hair follicles to the lower dermis, while some $\mathrm{N}$ Phsoms penetrate though the skin via intercellular and transcellular pathways. ${ }^{59}$ However, in P-Phsoms the fluorescence was localized at the hair root before being distributed to the viable upper layers. These results lined with that P-Phsoms was penetrated though hair follicles as the main penetration pathway. ${ }^{24}$

Regarding NLCs and AlgNPs, NLCs also showed relatively uniform distribution in epidermis and dermis with a high amount present in the SC $82.2 \pm 12.07$ Pixel/ $\mu \mathrm{m}^{2}$ (Figure 3A (c)) as N-Phsoms. Figure 3A (d) revealed 

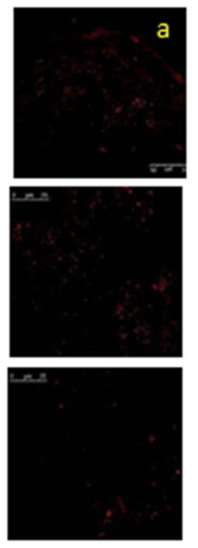

C
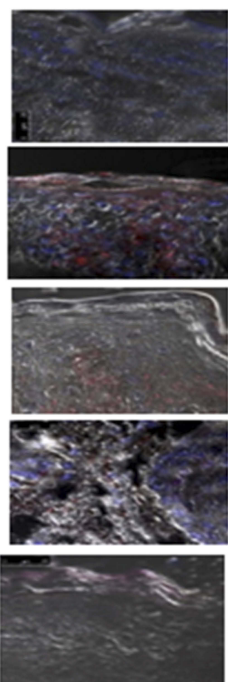
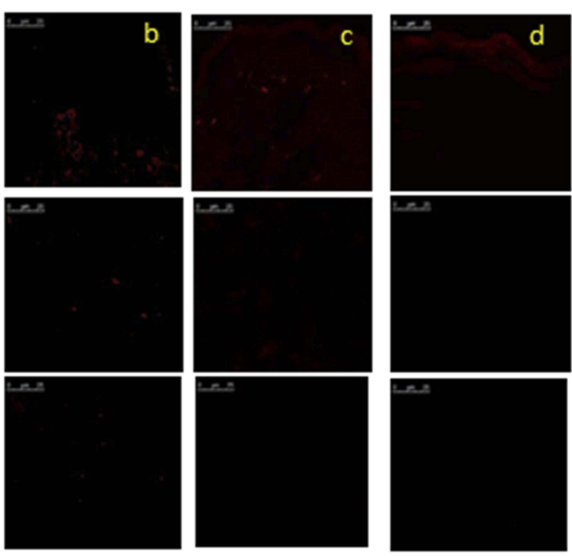

B

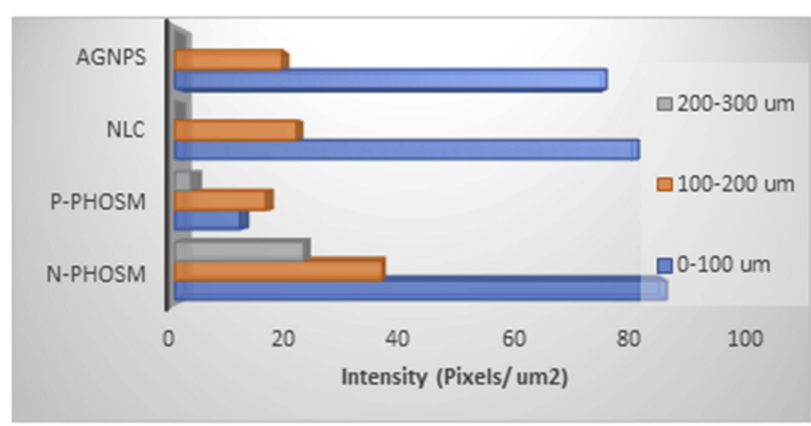

D
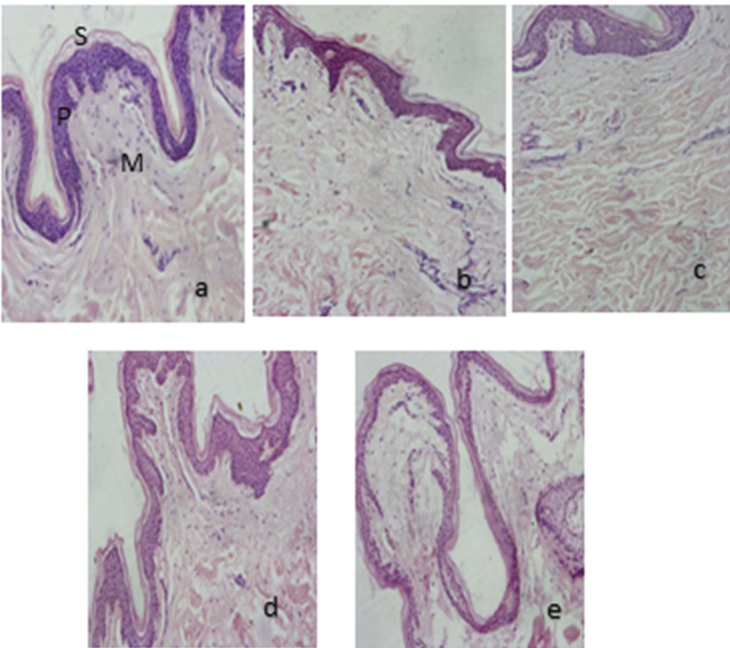

Figure 3 (A) CLSM images of a cross-section of human abdominal skin incubated on a Franz diffusion cell with different formulations containing ETD. The nanocarriers were applied for 6 hrs non-occlusively: (a) N-Phsoms, (b) P-Phsoms, (c) NLCs, and (d) AlgNPs. (B) Bar chart represents the fluorescence intensity (Pixels/ $\mu m^{2}$ ) of all nanoformulations at different depth intervals $\left(0-100,100-200\right.$, and 200-300 $\left.\mathrm{m}^{2}\right)$. (C) Overlay of blue fluorescence of DAPI, bright-field image, and red fluorescence of fluorescent dye (Rhodamine B). (D) Light microscopy images of human skin samples (stained with hematoxylin and eosin) after 24 hrs of treatment I\% ETD nanoformulations: (a) control group (untreated skin); (b) N-Phsoms; (c) P-Phsoms; (d) NLCs; and (e) AlgNPs. Letters S, P, and M refer to stratum corneum, epidermis, and dermis, respectively.

Abbreviations: NLCs, nanostructured lipid carriers; AlgNPs, alginate nanoparticles; CLSM, confocal laser scanning microscopy; ETD, etodolac; St.c, stratum corneum; DAPI, 4, 6-diamidino-2-phenylindole.

relatively low intensity of AlgNPs at the dermal layers, but a very strong intensity on the St.C and epidermal layer $\left(76.5 \pm 2.69 \mathrm{Pixel} / \mu \mathrm{m}^{2}\right)$. At deeper skin layers (200-300 $\mu \mathrm{m})$ both NLCs and AlgNPs recorded almost no intensity. Since the experiment last for $6 \mathrm{hrs}$, NLCs as described before by G. Chenyu et al, ${ }^{60}$ were in the stage of interaction with St.C forming adhesive layer that could subsequently increase skin hydration and drug penetration. Our previous finding about AlgNPs skin permeation was confirmed with the CLSM micrographs of the distribution, and localization of AlgNPs in the epidermal layers. This finding was also augmented by $\mathrm{H} \mathrm{T} \mathrm{P}$
Nguyen et al, ${ }^{26}$ where the fluorescence of curcumin was located in the first $50 \mu \mathrm{m}$ of skin with weak fluorescence seen in deeper skin make it suitable for the treatment of superficial skin diseases.

Overlay of H\&E images on DAPI stained images (Figure 3C) cleared the presence of fluorescence around the blue DAPI dye of the cellular nuclear, emphasizing intercellular pathway and allocation of the dye constituted in both P- and N-Phsoms and NLCs. However, AlgNPs mainly present in the dead uppermost St.C. Our results were compatible and closely correlated to ex-vivo permeation and deposition study. N-Phsoms accumulated the most 
in epidermis and dermis layers, but on the other hand NLCs and AlgNPs were mostly accumulated in St.C, imparting good correlation. As reported in the literature, SC primarily initiates in epidermis of the skin. Forming abnormal growth of cells that destroys basement membrane and invade the dermis. Therefore, for the more reliable treatment outcomes, the drug should be retained between epidermis and dermis of skin. ${ }^{61}$

\section{Assessment of skin irritation potential}

All the investigated nanocarriers were subjected to the skin irritation study to evaluate their safety. Insignificant histological differences between normal skin (negative control), and treated skin with $\mathrm{N}$ - and P-Phsoms (Figure 3D $(\mathrm{a}-\mathrm{c})$. As obvious in the images, a well-stratified epidermis, complete restoration of St.C. layers (St.C, stratum granulosum, stratum spinosum), and dermal layers with no signs of irritation were inspected visually and microscopically. This observation could be related to the hydration originated from the liposomal dispersion over the skin, that did not impact on its integrity. ${ }^{62}$ On the other hand, skin treated with NLCs and AlgNPs (Figure 3D (d and e) showed some signs of inflammation. NLCs showed a case of parakeratosis, which is an increased thickness of epidermis with multiple nuclei and increased intracellular space. However, AlgNPs showed hyper cellularity with an intraepidermal cellular infiltration. ${ }^{60}$

\section{In-vitro anticancer activity}

The anticancer activity of ETD-nanocarriers on SCC-9 SC cells was evaluated by MTT assay and compared to ETD solution. The in-vitro results showed that ETD expressed significant increased cell growth inhibition as dose increased after $24 \mathrm{hrs}$ in a dose-dependent manner.

The results revealed that all formulations were able to inhibit cell proliferation and \% cell growth inhibition was increased on increasing ETD concentration (Figure 4A). Moreover, the $\mathrm{IC}_{50}$ values of free ETD on SCC-9 cells were determined to be $982.75 \mu \mathrm{g} / \mathrm{mL}$ that is significantly higher than nanoformulations. N-Phsoms, P-Phsoms, NLCs, and AlgNPs demonstrated a significant decrease in cell viability by $5.40,4.33,1.85,1.64$-fold, respectively compared to free ETD $(P<0.01)$ as summarized in Table 4. The difference in the $\mathrm{IC}_{50}$ values compared to free ETD may be attributed to encapsulation of ETD in nanometric carrier that subsequently increased ETD cellular internalization by endocytosis or phagocytosis, resulting in sustained exposure of cancer cell to the drug. However, in free ETD cellular uptake and internalization depended mainly on time-limited diffusion mechanism which decreased by time due to intracellular saturation. The more effective treatment may be also related to the better solubility of ETD after being loaded into nanoformulations. ${ }^{49,63}$ Precisely, the main mechanism by which NSAIDs inhibit tumorigenesis is not well known. It may be attributed to inhibition of arachidonic acid metabolism and prostaglandin production. Inhibition of $\mathrm{COX}-2$ was reported to suppresses adenoma development in APC gene mode by suppressing the formation of $\beta$-catenin/TCF complexes and suppressing cell proliferation. In addition, COX-2 gene overexpression might resulted in changes in cellular adhesion (inactivation of E-cadherin) and inhibition of apoptosis. Caco2 cells growth suppression by ETD was found to be associated with upregulation of E-cadherin mRNA and protein. ${ }^{64}$ Since, ETD-N-Phsoms demonstrated the highest cytotoxicity activity on SCC-9 cells among other formulations and based on its higher deposition in the viable skin layer. These nanovesicles were selected for further studies.

\section{Wound-healing assay}

The in-vitro wound-healing scratch assay is a simple and economical method to study the cell migration activity. It has the advantage of mimicking to a certain extent the invivo behavior of tumoral cells during migration. Current assay is based on creation of a scratch, which is an artificial gap between cells on a confluent cells monolayer. Cells on both edges will migrate to close the created gap and to return cell-cell contact. Images were captured at the beginning 0 time and after 24 hrs during cell migration process to close the scratch. Comparison of the both images to determine \% cell migration inhibition. ${ }^{65,66}$

Our study is aligned to investigate whether encapsulating ETD in a nanocarrier played a role in preventing tumor metastasis. Accordingly, migration property of SCC was studied. Pictures were taken at 0 and $24 \mathrm{hrs}$ (Figure 4B) and the $\%$ cell migration inhibition is depicted in Figure $4 \mathrm{C}$. Untreated cells revealed recovery of the wounded area (Figure 4B (a, d)) with about 40\% inhibition. The in-vitro migration activity of SCC cells was noticeably reduced by treatment with free ETD (Figure 4B (b, e)), which markedly decreased with the use of nanocarrier (Figure 4B (c, f)). A significant difference in $\%$ cell migration inhibition was obtained from Free ETD (55\%) and ETD-nanocarrier $(80 \%$,). This result obviously indicates the activity of ETD to inhibit the motility of SCC cells that significantly 

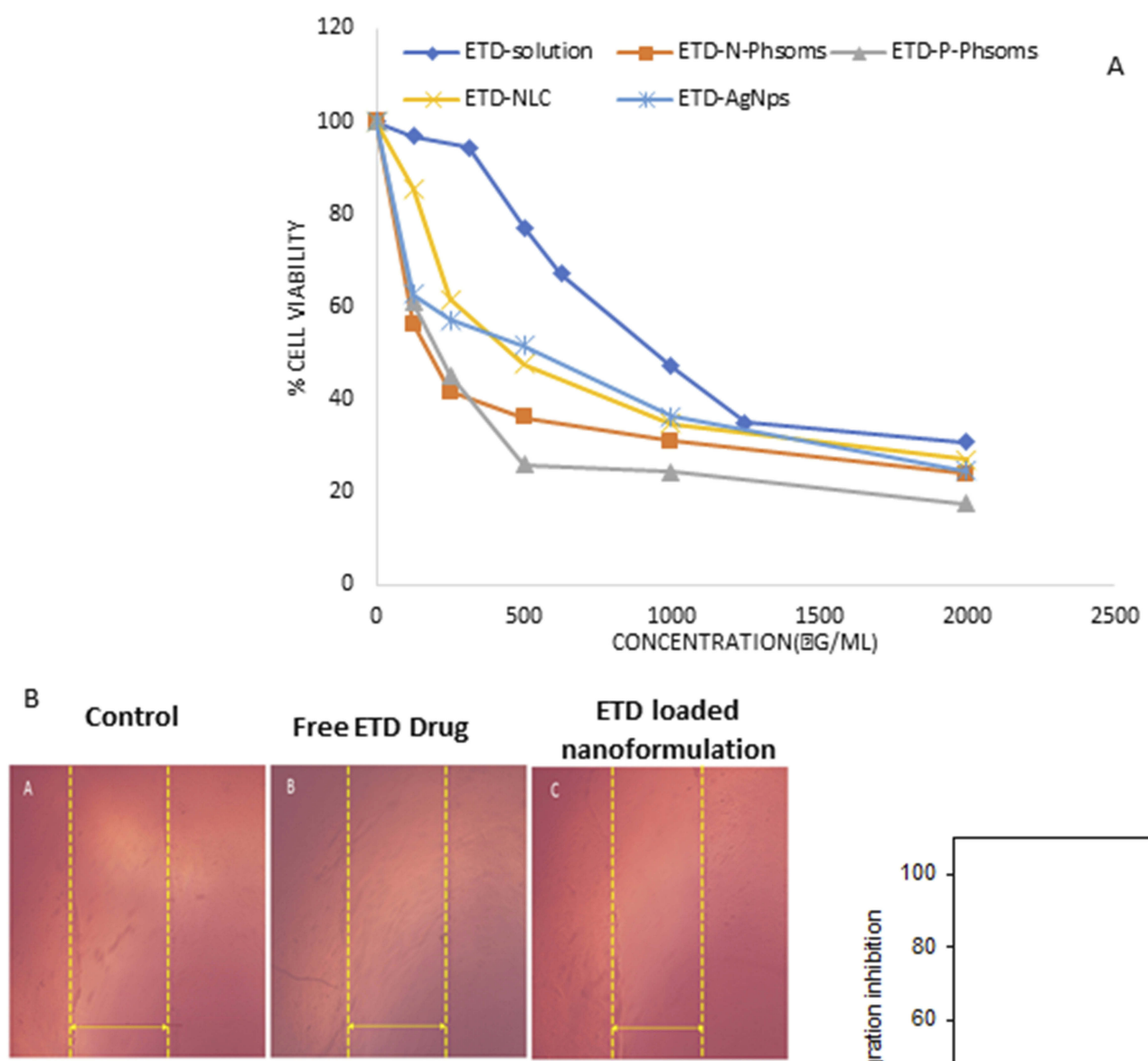

\section{Free ETD Drug}

ETD loaded nanoformulation
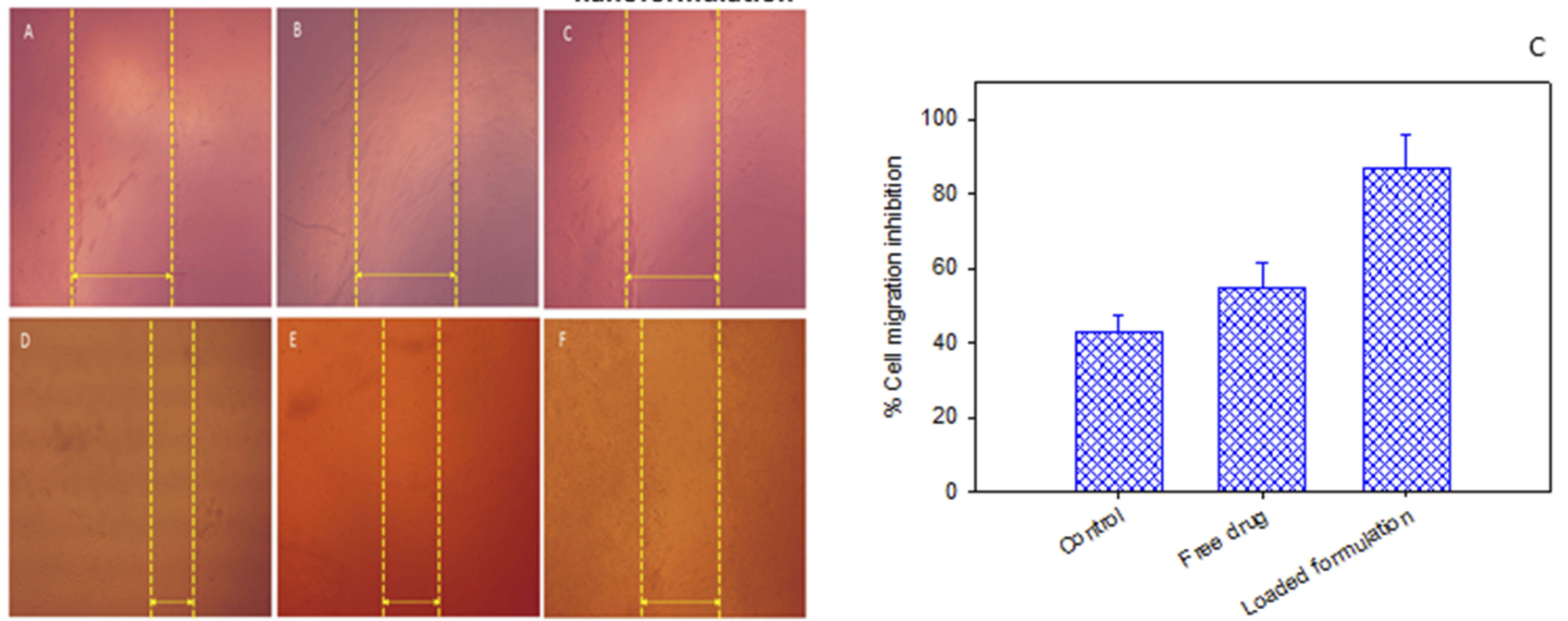

Figure 4 (A) Percent viability of SCC-9 skin cancer cell measured by MTT cytotoxicity assay after exposure to ascending concentrations of free ETD solution, ETDloaded N-Phsoms, P-Phsoms, NLCs, and AlgNPs for 24 hrs, data were shown as mean \pm SD ( $n=3$ ), $P<0.05$. (B) Images acquired during a wound-healing experiment, (parts A\&D) are for control untreated SCC, (parts B\&E) are for SCC treated with Free ETD drug, (parts C\&F) for SCC treated with ETD-loaded N-Phsoms formulation at the time $\mathrm{t}=0$ and $\mathrm{T}=24 \mathrm{hrs}$. (C) Bar chart of \% cell migration inhibition of control untreated SCC, SCC treated with Free ETD drug, and ETD- loaded N-Phsoms formulation after $24 \mathrm{hrs}$.

Abbreviations: ETD, etodolac; NLCs, nanostructured lipid carriers; AlgNPs, alginate nanoparticles; SCC, squamous cell carcinoma.

Table $4 \mathrm{IC}_{50}$ values of ETD-loaded N-Phsoms, P-Phsoms, NLCs, and AlgNPs compared to free ETD solution on SCC-9 skin cancer cells after $24 \mathrm{hrs}$

\begin{tabular}{|l|l|}
\hline Formulation & IC $_{\mathbf{5 0}}$ \\
\hline Free ETD solution & 982.75 \\
ETD-N-Phsoms & 181.76 \\
ETD-P-Phsoms & 227.11 \\
ETD-NLCs & 529.52 \\
ETD-AlgNPs & 597.86 \\
\hline
\end{tabular}

Abbreviations: ETD, etodolac; NLCs, nanostructured lipid carriers; AlgNPs, alginate nanoparticles. increased after incorporation in lipid nanocarrier $(P<0.005)$ as a result of enhanced cell internalization by the nanocarrier. As reported previously, nanocarriers may exhibit their effect by preventing and/or delaying the dissemination of viable cells from the primary tumor rather than by exerting a direct cytotoxic influence on micro-metastasis. ${ }^{23}$ According to the reported literature, COX-2 makes cancerous cells resistant to apoptosis and promotes cell metastasis. Since, COX-2 upregulate vascular endothelial human growth factor $\mathrm{C}$ (VEGF-C). VEGF-C and COX-2 
expressions work together to enhance peritumoral lymph angiogenesis. Excessive lymphatic vessel formation has implicated in neoplasm metastasis. COX-2 inhibition results in an inhibition of peritoneal metastasis by gastric cancer cells, lymph node metastasis in oral SCC, and liver metastases of colon cancer. ${ }^{7,67,68}$ Moreover, it was found that COX-2 overexpression is accompanied by matrix metalloproteinases (MMPs), particularly, MMP-2 and MMP-9. They play a crucial role in the migration of malignancies process as they involve in degradation of extracellular matrix and basement membrane. Hence, inhibition of MMP2/9 by COX-2 inhibitor such as celecoxib was found to suppress cancerous cell migration and invasion. ${ }^{36}$

\section{In-vivo chemoprotective potential}

EAC was adopted in the current study as it could be induced as ascites or as a solid tumor form. EAC is easy to grow in suspension in the peritoneal cavity of mice. Further, EAC suspension contains homogeneous free tumor cells so it has a transplantable capacity to another mouse. Lastly, EAC cell line is easily prepared, grown and safe model for in-vivo experiments. ${ }^{69-71}$

\section{Toxicity study}

Drug safety has superior priority in drug evaluation process. In this regard, animals' body weight (wt) percent change was recorded during study period (Figure 5A). Firstly, no signs of dermal toxicity (eg, erythema) was visually observed during the course treatment. Moreover, no mortality was occurred during the experiment. It was observed that mice treated with ETD nano appeared healthy with a significant increase in their body wt and $\%$ body wt change compared to free ETD and positive control $(P<0.005)$. The body wt \% increase was 12.15 $\pm 2.16 \%$, while the body weight change in the free ETD-treated group and positive group was $1.60 \pm 2.38$, and $0.23 \pm 1.71 \%$, respectively. This result may be referred to the cumulative toxicity of free ETD compared to ETD encapsulated in nanoformulation. Further clinical evaluation of body systems, organs, and organs' weight ratio revealed, no significant difference in liver or kidney relative weight (Figure 5B). On the contrary, there was a significantly higher spleen relative weight in free ETDtreated and positive groups $(P=0.001)$ by 1.8 and 2.33 -fold increase compared to negative control. ETD nano revealed normalized spleen wt ratio assured its greatest anti-tumor effect as demonstrated previously by Rivenson. A. et al, ${ }^{72}$ that splenomegaly in Ehrlich-bearing mice is a marked indication on the presence of the tumor in its solid state. Since, some excipients such as Tween 80 are reported to be cytotoxic to the kidney. Assessment of any potential systemic toxicity of ETD and excipients used was assessed by evaluating liver and kidney functions. No significant hepatotoxic or nephrotoxic effects compared with negative control were found as recorded in Table 5. The latter results revealed the role of ETD encapsulation in nanocarrier (ETD-N-Phsoms) to decrease the effect of ETD treatment on their body weights with a good safety profile.

\section{Efficacy study}

Encapsulation of ETD in nanoformulation compiled a significant $(P<0.05)$ reduction in relative tumor weight (Figure 5C) compared to free ETD and positive control. ETD nano depicted a relative tumor wt of $480 \pm 0.02 \mathrm{mg}$ compared to $820 \pm 0.04$ and $720 \pm 0.04 \mathrm{mg}$ in case of positive control and free ETD-treated groups, respectively.

Since ETD is a COX-2 inhibitor, its tumor progression inhibition is proceeded through the inhibition of various proinflammatory and proliferation factors. Activity of ETD on COX-2 mRNA and PCNA mRNA expression was assessed. As reported COX-2-selective inhibitors have been shown to induce apoptosis in a wide variety of solid tumor cell lines, such as glioma, head and neck, cervical, and colon cancer, through activation of caspases 3 by COX-2 dependent and independent cell growth inhibition. ${ }^{12}$ In the current study, ETD nano demonstrated the highest significant tumor growth inhibition $(P<0.05)$ with a reduction in relative tumor wt by 1.70 and 1.51-fold compared to positive control and free ETD, respectively. ETD nano exhibited 1.72-fold reduction in both COX-2 and PCNA mRNA levels (Figure 5D and E) and 2.63-fold elevation in caspase-3 level in skin tumors relative to the positive control group (Figure 5F). Comparatively, the group treated with free ETD has shown merely 1.06-fold reduction both COX-2 and PCNA mRNA levels and 1.57-fold elevation of caspase-3 relative to the positive control $(P<0.05)$. COX-2 mRNA elevated levels were expressed in precancerous lesions in the human head and neck region. ${ }^{17}$ The obtained results clearly elucidated that ETD nanoformulations exhibited a considerably higher survival rate, chemopreventive activity, apoptotic, and antiangiogenic activities which resulted in superior therapeutic effects compared with free ETD. The superiority in in-vivo chemopreventive efficacy was also supported by the results of in-vitro cytotoxicity study, where ETD-loaded N-Phsoms showed higher cytotoxic potential due to their higher cellular internalization than that of free ETD after $24 \mathrm{hrs}$. The nano- 

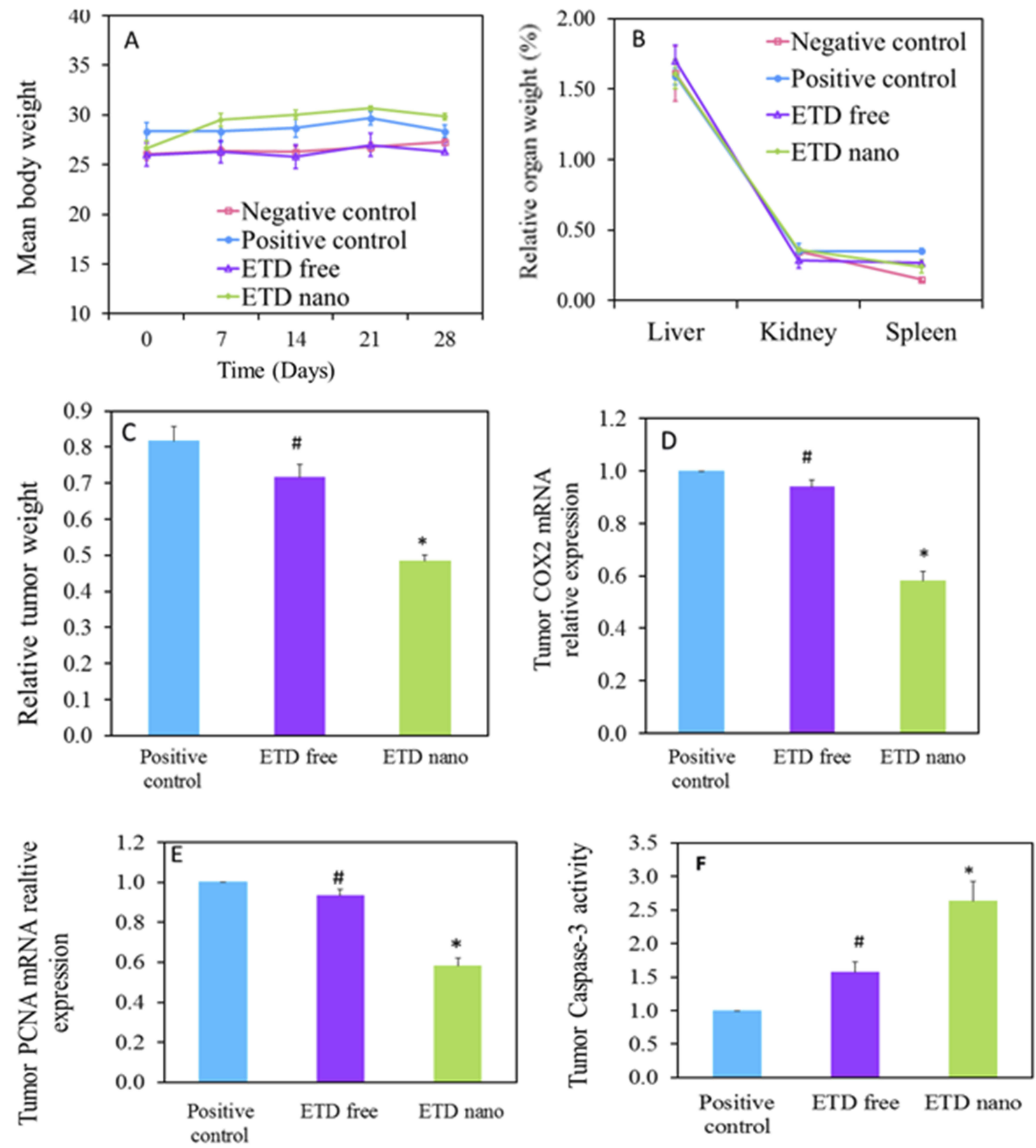

Figure 5 (A) Animals' body weight measurements (g) during ETD treatment. (B) Animals' relative organ weight (\%) after ETD treatment. (C) Relative tumor weight after ETD treatment. (D) Effect of ETD on tumor COX-2 mRNA expression. (E) Effect of ETD on tumor PCNA mRNA expression. (F) Effect of ETD on tumor Caspase 3 activity. ${ }^{*} P<0.05$ vs positive control, ${ }^{*} P<0.05$ vs ETD nano.

Abbreviations: ETD, etodolac; PCNA, proliferating cell nuclear antigen.

scaled size enables the particles to exploit the porosity of tumor vasculature and pass directly to tumoral cells. Furthermore, it provides protection for drug molecules from interactions with other biological components with subsequent improved intratumor drug stability, controlled drug release, and modified pharmacokinetics and biodistribution. ${ }^{23}$ The aforementioned results were further augmented with histopathological examination.

\section{Histopathological and IHC examination}

In the light of the previous biochemical analysis, both free ETD and ETD nano visually revealed a marked decrease in the tumor volume and tumor scar compared to positive control group (Figure 6A and B). Consequently, the histological examination of the excised tumors from ETD nano and free ETD-treated groups in comparison with positive and negative groups was elaborated (Figure 6C). 
Table 5 Effect of ETD treatment on serum liver and kidney functions

\begin{tabular}{|l|l|l|l|l|}
\hline & ALT (U/L) & AST (U/L) & Urea (mg/dL) & Creatinine (mg/dL) \\
\hline Negative control & $29.67 \pm 0.33$ & $27.33 \pm 1.38$ & $24.52 \pm 1.0$ & $0.78 \pm 0.06$ \\
Positive control & $29.33 \pm 0.71$ & $26.0 \pm 1.81$ & $22.18 \pm 0.35$ & $0.77 \pm 0.06$ \\
ETD free & $29.67 \pm 0.49$ & $24.50 \pm 0.85$ & $23.35 \pm 1.16$ & $0.78 \pm 0.06$ \\
ETD Nano & $29.83 \pm 0.54$ & $24.83 \pm 1.66$ & $23.45 \pm 1.03$ & $0.78 \pm 0.06$ \\
\hline
\end{tabular}

Abbreviations: ETD, etodolac; ALT, Alanine aminotransferase; AST, aspartate aminotransferase.

A
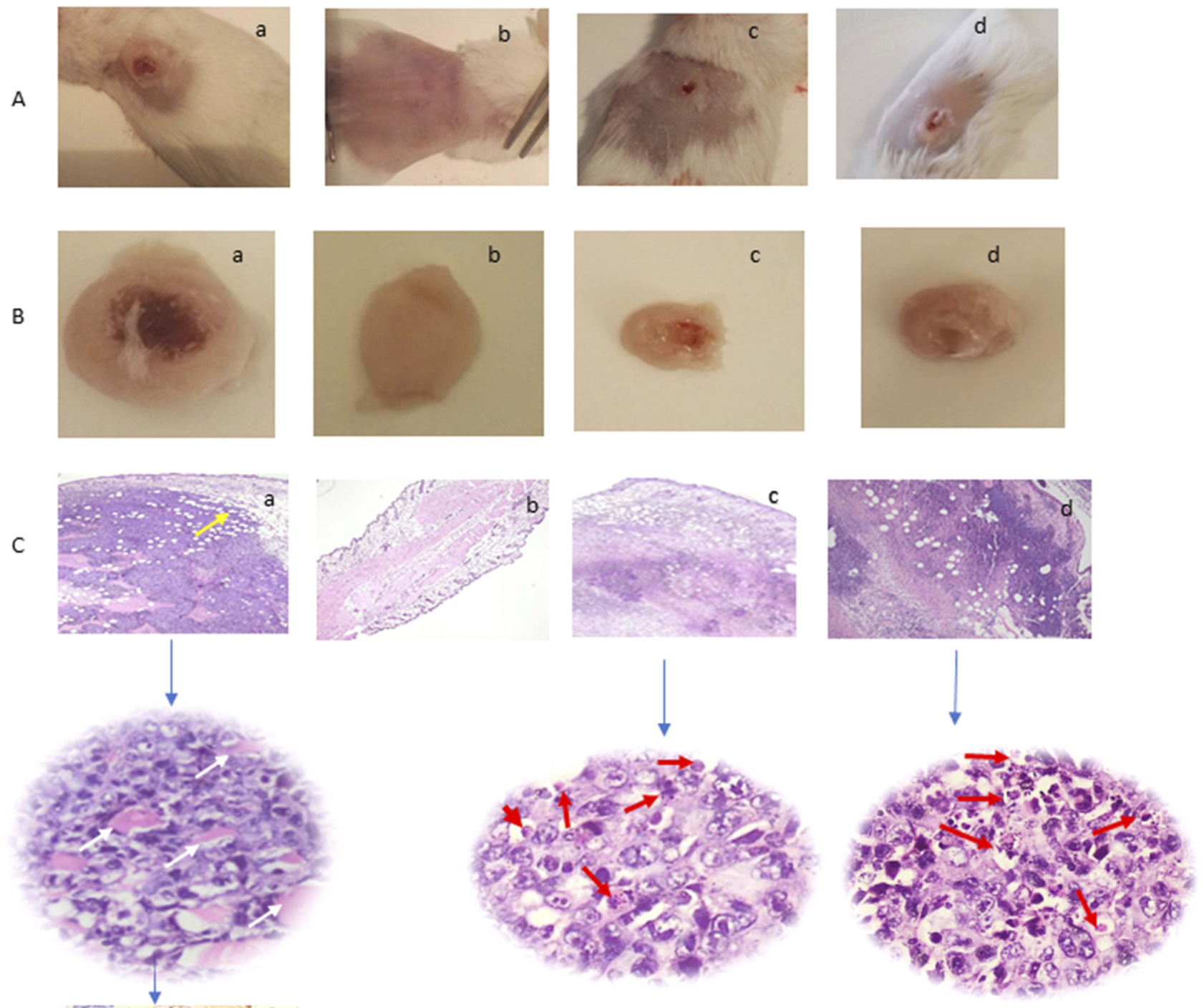

D

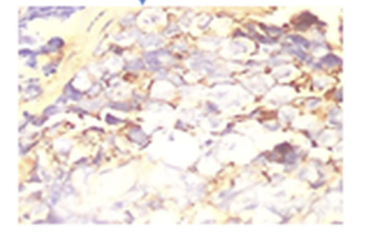

Figure 6 (A) Tumor-bearing mice. (B) Excised tumors. (C) H\&E histopathological examination of tumor tissue sections at the end of study period using two magnification powers $\times 200$ and 400 original magnification. (D) Immunohistochemical staining of tumor tissue shows focal and strong positive staining of the malignant cells to Multi-Cytokeratin AEI/AE3 (Ready to use primary antibody, Mouse anti-human, monoclonal antibody, P00I2, Bond-Max fully automated immunostainer, Leica Biosystems, USA). For the following groups, (a) untreated (positive control) tumor infiltrating the dermis, subcutaneous, and muscular tissues (yellow arrow). The tumor is formed of masses of malignant cells with pleomorphism, hyperchromatism, vesicular nuclei, and prominent nucleoli with frequent tumor giant cells (white arrow), (b) Normal (Negative control), (c) ETD Nanoformulation treated (red arrows point to apoptotic cells and apoptotic debris), and (d) ETD solution treated (red arrows point to apoptotic cells and apoptotic debris).

Abbreviations: H\&E, hematoxylin and eosin; ETD, etodolac. 
IHC staining of the tumor tissue (Figure 6D) shows the malignant cells focally positive to Multi -Cytokeratin AE1/AE3, while negative to HMB-45. The lack of tumor differentiation by H\&E and the IHC-staining pattern favor the diagnosis of undifferentiated carcinoma of the skin.

The histopathological examination of skin biopsy from group II (positive control) using H\&E (Figure 6C (a)) in comparison to normal skin (Figure 6C (b)) featuring a malignant tumor infiltrating the dermis, subcutaneous, and muscular tissues (yellow arrow). The tumor is formed of masses of malignant cells with severe atypical changes in the form of cellular and nuclear pleomorphism, hyperchromatism, increased nuclear/cytoplasmic ratio, vesicular nuclei, prominent nucleoli, brisk mitotic figures with many abnormal forms and frequent tumor giant cells (white arrow, $\times 400$ original magnification) and wide areas of necrosis. The overlying epidermis shows focal areas of dysplastic changes with cytological atypia. The tumor shows no specific differentiation. In comparison to the normal skin without tumorous growth (Figure 6C (b), $\times 200$ original magnification).

On the other hand, tissue sections treated by ETD nano (Figure 6C (c)) revealed a decrease in the tumor mass with residual tumor of $15 \%$, with wide areas of necrosis. Many apoptotic cells were identified as condensed nuclei and cytoplasm as well as apoptotic bodies and debris within the residual tumor mass identifying the drug-induced apoptosis (red arrows, Figure 6C (c), $\times 400$ original magnification). The same features were found by the histopathological assessment of the tumors treated by free ETD (Figure 6C (d)) in which the tumor mass decreased with residual tumor of $20 \%$ with wide areas of necrosis. Frequent apoptotic cells and apoptotic bodies and debris were also identified within the residual tumor mass (represented by red arrows, (Figure 6C (d), H\&E, $\times 400$ original magnification) highlighting the drug-induced apoptotic effect.

The superior efficacy of ETD nanoformulation could be attributed to its enhanced penetration and retention within the tumor. The nanometric formulation with 202 $\mathrm{nm}$ can easily penetrate the tumor vascular system combined with the tumor vessels' leaky nature and its ineffective drainage systems, enhancing its retention. Thus, provide an evidence on that incorporation of ETD in NPhsoms is a promising candidate as a chemopreventive agent against SCC. ${ }^{17}$

\section{Conclusion}

The current piece of work highlights the importance of the nanocarrier selection for a drug to achieve the desired and planned attributes of safety, and efficacy. As far as ETD is concerned, for chemoprevention of SC, better penetrating and retaining carriers like. N-Phsoms NLCs and AlgNPs can perform better than P-Phsoms. Appreciable dermal compliance can be achieved with Phosalosomal formulations. All nanocarriers exhibited invitro cytotoxic activity against $\mathrm{SCC}$, with $\mathrm{IC}_{50}$ values lower than free ETD. Incorporation in nanocarrier increased ETD in-vitro migration activity inhibition. Inhibition of COX-2 has implicated in VEGF-C upregulation and tumor metastasis. Chemopreventive activity of ETD was also promoted upon loading in nanocarrier. It showed significant inhibition of COX-2 mRNA expression, and PCNA mRNA expression, with enhanced Caspase-3 activity. Former significant results reflected a significant decrease in tumor volume, combined with perfect safety profile on gross and organ level. These findings can be extrapolated to several other similar drugs.

\section{Acknowledgments}

The authors acknowledge the Department of Industrial Pharmacy and Pharmaceutical Nanotechnology, Faculty of Pharmacy, Alexandria University for the provided support for the purchase of chemicals and running the in-vitro experiments. Further acknowledgment goes to the Centre of Excellence for Research in Regenerative Medicine and its Applications (CERRMA) at Faculty of Medicine, Alexandria University, Egypt, for running and analysis of confocal laser and optical images, Molecular Biology and Nanomedicine Labs for in-vivo biochemical tests. The authors also acknowledge the Medical Research Centre at the Faculty of Medicine, Alexandria University, Egypt where cell culture and in-vivo experiments were conducted.

\section{Disclosure}

The authors report no personal or financial conflicts of interest regarding this paper.

\section{References}

1. Ijaz S, Akhtar N, Khan MS, et al. Plant derived anticancer agents: a green approach towards skin cancers. Biomed Pharmacother. 2018;103:1643-1651. doi:10.1016/j.biopha.2018.04.113 
2. Goyal R, Macri LK, Kaplan HM, Kohn J. Nanoparticles and nanofibers for topical drug delivery. J Controlled Release. 2016;240:77-92. doi:10.1016/j.jconrel.2015.10.049

3. Simoes MCF, Sousa JJS, Pais A. Skin cancer and new treatment perspectives: a review. Cancer Lett. 2015;357(1):8-42. doi:10.1016/ j.canlet.2014.11.001

4. Kono M, Watanabe M, Abukawa H, Hasegawa O, Satomi T, Chikazu D. Cyclo-oxygenase-2 expression is associated with vascular endothelial growth factor $\mathrm{C}$ expression and lymph node metastasis in oral squamous cell carcinoma. J Oral Maxillofacial Surg. 2013;71 (10):1694-1702. doi:10.1016/j.joms.2013.04.015

5. Su J, Shih J, Yen M, et al. Cyclooxygenase-2 induces EP1-and HER2/Neu-dependent vascular endothelial growth factor-C up-regulation: a novel mechanism of lymphangiogenesis in lung adenocarcinoma. Cancer Res. 2004;64(2):554-564.

6. Tendo M, Yashiro M, Nakazawa K, et al. A synergic inhibitory-effect of combination with selective cyclooxygenase-2 inhibitor and S-1 on the peritoneal metastasis for scirrhous gastric cancer cells. Cancer Lett. 2006;244(2):247-251. doi:10.1016/j.canlet.2005.12.019

7. Sorski L, Melamed R, Matzner P, et al. Reducing liver metastases of colon cancer in the context of extensive and minor surgeries through beta-adrenoceptors blockade and COX2 inhibition. Brain Behav Immun. 2016;58:91-98. doi:10.1016/j.bbi.2016.05.017

8. Kapadia GJ, Azuine MA, Shigeta Y, Suzuki N, Tokuda H. Chemopreventive activities of etodolac and oxyphenbutazone against mouse skin carcinogenesis. Bioorg Med Chem Lett. 2010;20 (8):2546-2548. doi:10.1016/j.bmcl.2010.02.093

9. Harris RE, Alshafie GA, Abou-Issa H, Seibert K. Chemoprevention of breast cancer in rats by celecoxib, a cyclooxygenase 2 inhibitor Cancer Res. 2000;60(8):2101-2103.

10. Reddy BS, Hirose Y, Lubet R, et al. Chemoprevention of colon cancer by specific cyclooxygenase- 2 inhibitor, celecoxib, administered during different stages of carcinogenesis. Cancer Res. 2000;60 (2):293-297.

11. Lin J, Hsiao P, Chiu T, Chao J. Combination of cyclooxygenase-2 inhibitors and oxaliplatin increases the growth inhibition and death in human colon cancer cells. Biochem Pharmacol. 2005;70(5):658-667. doi:10.1016/j.bcp.2005.05.028

12. Shogo M, Adachi M, Kioi M, Torigoe S, Ijichi K, Hasegawa Y, et al. Etodolac improves 5-FU sensitivity of head and neck cancer cells through inhibition of thymidylate synthase. Anticancer Res. 2011;31:2893-2898.

13. Ratnasinghe D, Daschner PJ, Anver MR, et al. Cyclooxygenase-2, Pglycoprotein-170 and drug resistance; is chemoprevention against multidrug resistance possible? Anticancer Res. 2001;21(3C):21412147.

14. Hasegawa K, Ishikawa K, Kawai S, et al. Overcoming paclitaxel resistance in uterine endometrial cancer using a COX-2 inhibitor. Oncol Rep. 2013;30(6):2937-2944. doi:10.3892/or.2013.2790

15. Lúcio M, Ferreira H, Lima J, Reis S. Use of liposomes as membrane models to evaluate the contribution of drug-membrane interactions to antioxidant properties of etodolac. Redox Rep. 2013;13(5):225-236. doi:10.1179/135100008X308939

16. Goindi S, Kaur R, Kaur R. An ionic liquid-in-water microemulsion as a potential carrier for topical delivery of poorly water soluble drug: development, ex-vivo and in-vivo evaluation. Int J Pharm. 2015;495 (2):913-923. doi:10.1016/j.ijpharm.2015.09.066

17. Yamamoto K, Kitayama W, Denda A, et al. Suppressive effects of a selective cyclooxygenase-2 inhibitor, etodolac, on 4-nitroquinoline 1oxide-induced rat tongue carcinogenesis. Exp Toxicol Pathol. 2004;56(3):145-151. doi:10.1016/j.etp.2004.07.001

18. de Souza Thiago L, Da Costa ES, Lopes DV, Borojevic R. Racemic etodolac is cytotoxic and cytostatic for B-cell precursor acute lymphoblastic leukemia cells. Biomed Pharmacother. 2009;63(7):548551. doi:10.1016/j.biopha.2008.09.009
19. Okamoto A, Shirakawa T, Bito T, et al. Etodolac, a selective cyclooxygenase-2 inhibitor, induces upregulation of E-cadherin and has antitumor effect on human bladder cancer cells in vitro and in vivo. Urology. 2008;71(1):156-160. doi:10.1016/j.urology.2007.09.061

20. Magari H, Shimizu Y, Inada K, et al. Inhibitory effect of etodolac, a selective cyclooxygenase-2 inhibitor, on stomach carcinogenesis in Helicobacter pylori-infected Mongolian gerbils. Biochem Biophys Res Commun. 2005;334(2):606-612. doi:10.1016/j.bbrc.2005.06.132

21. Mishima K, Nariai Y, Yoshimura Y. Etodolac, a selective cyclo-oxygenase-2 inhibitor, enhances carboplatin-induced apoptosis of human tongue carcinoma cells by down-regulation of FAP-1 expression. Oral Oncol. 2005;41(1):77-81. doi:10.1016/j.oraloncology.2004.06.009

22. Boakye CHA, Patel K, Doddapaneni R, et al. Ultra-flexible nanocarriers for enhanced topical delivery of a highly lipophilic antioxidative molecule for skin cancer chemoprevention. Colloids Surf B Biointerfaces. 2016;143:156-167. doi:10.1016/j.colsurfb.2016.03.036

23. Fernandes RS, Silva JO, Monteiro LOF, et al. Doxorubicin-loaded nanocarriers: a comparative study of liposome and nanostructured lipid carrier as alternatives for cancer therapy. Biomed Pharmacother. 2016;84:252-257. doi:10.1016/j.biopha.2016.09.032

24. Boakye CH, Patel K, Singh M. Doxorubicin liposomes as an investigative model to study the skin permeation of nanocarriers. Int $J$ Pharm. 2015;489(1-2):106-116. doi:10.1016/j.ijpharm.2015.04.059

25. Oskuie AB, Nasrollahi S, Nafisi S. Design, synthesis of novel vesicular systems using turpentine as a skin permeation enhancer. J Drug Deliv Sci Technol. 2018;43:327-332. doi:10.1016/j.jddst.2017.10.015

26. Nguyen H, Munnier E, Soucé M, et al. Novel alginate-based nanocarriers as a strategy to include high concentrations of hydrophobic compounds in hydrogels for topical application. Nanotechnology. 2015;26(25):255101. doi:10.1088/0957-4484/26/25/255101

27. Gupta M, Goyal AK, Paliwal SR, et al. Development and characterization of effective topical liposomal system for localized treatment of cutaneous candidiasis. J Liposome Res. 2010;20(4):341-350. doi:10.3109/08982101003596125

28. Sarmento B, Ferreira D, Veiga F, Ribeiro A. Characterization of insulin-loaded alginate nanoparticles produced by ionotropic pregelation through DSC and FTIR studies. Carbohydr Polym. 2006;66(1):1-7. doi:10.1016/j.carbpol.2006.02.008

29. Grillo R, de Melo NF, de Araujo DR, de Paula E, Rosa AH, Fraceto LF. Polymeric alginate nanoparticles containing the local anesthetic bupivacaine. J Drug Target. 2010;18(9):688-699. doi:10.3109/ 10611861003649738

30. Barakat NS. Etodolac-liquid-filled dispersion into hard gelatin capsules: an approach to improve dissolution and stability of etodolac formulation. Drug Dev Ind Pharm. 2006;32(7):865-876. doi:10.1080/03639040500534192

31. Sallam MA, Motawaa AM, Mortada SM. An insight on human skin penetration of diflunisal: lipogel versus hydrogel microemulsion. Drug Dev Ind Pharm. 2015;41(1):141-147. doi:10.3109/03639045. 2013.850711

32. Sallam MA, Boscá M. Mechanistic analysis of human skin distribution and follicular targeting of adapalene loaded biodegradable nanospheres with an insight into hydrogel matrix influence, in-vitro skin irritation and in-vivo tolerability. J Pharm Sci. 2017. doi:10.1016/j. xphs.2017.05.038

33. Mohyeldin SM, Mehanna MM, Elgindy NA. Superiority of liquid crystalline cubic nanocarriers as hormonal transdermal vehicle: comparative human skin permeation-supported evidence. Expert Opin Drug Deliv. 2016;13(8):1049-1064. doi:10.1080/17425247.2016.1182490

34. Shankar S, Pangeni R, Park J, Rhim J. Preparation of sulfur nanoparticles and their antibacterial activity and cytotoxic effect. Mater Sci Eng. 2018. doi:10.1016/j.msec.2018.07.015

35. Gibadullina NN, Latypova DR, Vakhitov VA, et al. Synthesis and cytotoxic activities of difluoroacetyl-substituted hexahydropyrimidine derivatives. J Fluor Chem. 2018;211:94-99. doi:10.1016/j. jfluchem.2018.04.011 
36. Li -W-W, Long G-X, Liu D-B, et al. Cyclooxygenase-2 inhibitor celecoxib suppresses invasion and migration of nasopharyngeal carcinoma cell lines through a decrease in matrix metalloproteinase-2 and -9 activity. Pharmazie. 2014;69:132-137.

37. Lazarus H, Tegeler W, Mazzone HM, Leroy JG, Boone BA, Foley GE. Determination of sensitivity of individual biopsy specimens to potential inhibitory agents: evaluation of some explant culture methods as assay systems. Cancer Chemother Rep. 1966;50(8):543-555.

38. Abd-Alhaseeb MM, Zaitone SA, Abou-El-Ela SHM, Moustafa YM. Olmesartan potentiates the anti-angiogenic effect of sorafenib in mice bearing Ehrlich's ascites carcinoma: role of angiotensin (1-7). PLoS One. 2014;9(1):e85891. doi:10.1371/journal.pone.0085891

39. Hsu SM, Raine L, Fanger H. Use of avidin-biotin-peroxidase complex $(\mathrm{ABC})$ in immunoperoxidase techniques: a comparison between $\mathrm{ABC}$ and unlabeled antibody (PAP) procedures. $J$ Histochem Cytochem. 1981;29(4):577-580. doi:10.1177/29.4.6166661

40. Iqbal B, Ali J, Baboota S. Silymarin loaded nanostructured lipid carrier: from design and dermatokinetic study to mechanistic analysis of epidermal drug deposition enhancement. $J$ Mol Liq. 2018;255:513-529. doi:10.1016/j.molliq.2018.01.141

41. Raza K, Singh B, Lohan S, et al. Nano-lipoidal carriers of tretinoin with enhanced percutaneous absorption, photostability, biocompatibility and anti-psoriatic activity. Int J Pharm. 2013;456(1):65-72. doi:10.1016/j.jpharm.2013.08.019

42. Hoeller S, Sperger A, Valenta C. Lecithin based nanoemulsions: a comparative study of the influence of non-ionic surfactants and the cationic phytosphingosine on physicochemical behaviour and skin permeation. Int J Pharm. 2009;370(1-2):181-186. doi:10.1016/j. ijpharm.2008.11.014

43. Rocha KAD, Krawczyk-Santos AP, Andrade LM, et al. Voriconazoleloaded nanostructured lipid carriers (NLC) for drug delivery in deeper regions of the nail plate. Int J Pharm. 2017;531(1):292-298. doi:10.1016/j.ijpharm.2017.08.115

44. López-García R, Ganem-Rondero A. Solid lipid nanoparticles (SLN) and nanostructured lipid carriers (NLC): occlusive effect and penetration enhancement ability. J Cosmet Dermatological Sci Appl. 2015;5(02):62. doi:10.4236/jcdsa.2015.52008

45. Ma R, Levard C, Marinakos S, et al. Size-controlled dissolution of organic-coated silver nanoparticles. Environ Sci Technol. 2011;46 (2):752-759. doi:10.1021/es201686j

46. Anwar DM, Khattab SN, Helmy MW, et al. Lactobionic/folate dual-targeted amphiphilic maltodextrin-based micelles for targeted codelivery of sulfasalazine and resveratrol to hepatocellular carcinoma. Bioconjug Chem. 2018;29(9):3026-3041. doi:10.1021/acs. bioconjchem. 8b00428

47. Vijayakumar A, Baskaran R, Jang Y, Oh S, Yoo B. Quercetin-loaded solid lipid nanoparticle dispersion with improved physicochemical properties and cellular uptake. AAPS PharmSciTech. 2016;18(3):875883.

48. Pavan KC, Padmapreetha J. Formulation and in-vitro evaluation of gel containing ethosomes entrapped with etodolac. IJPSR. 2014;5:2.

49. Chen Y, Yang X, Zhao L, et al. Preparation and characterization of a nanostructured lipid carrier for a poorly soluble drug. Colloids Surf A. 2014;455:36-43. doi:10.1016/j.colsurfa.2014.04.032

50. Khampienga T, Aramwitb P, Supaphol P. Silk sericin loaded alginate nanoparticles: preparation and anti-inflammatory efficacy. Int J Biol Macromol. 2015;1(80):636-643.

51. Hans M, Lowman A. Biodegradable nanoparticles for drug delivery and targeting. Curr Opin Solid State Mater Sci. 2002;6(4):319-327. doi:10.1016/S1359-0286(02)00117-1

52. Chen H, Wang Y, Zhai Y, Zhai G, Wang Z, Liu J. Development of a ropivacaine-loaded nanostructured lipid carrier formulation for transdermal delivery. Colloids Surf A. 2015;465:130-136. doi:10.1016/j. colsurfa.2014.10.046
53. Katare O, Raza K, Singh B, Dogra S. Novel drug delivery systems in topical treatment of psoriasis: rigors and vigors. Indian J Dermatol Venereol Leprol. 2010;76(6):612. doi:10.4103/0378-6323.72451

54. Contri RV, Fiel LA, Alnasif N, Pohlmann AR, Guterres SS, SchaferKorting M. Skin penetration and dermal tolerability of acrylic nanocapsules: influence of the surface charge and a chitosan gel used as vehicle. Int $J$ Pharm. 2016;507(1-2):12-20. doi:10.1016/j.ijpharm. 2016.03.046

55. Gillet $\mathrm{A}$, Compère $\mathrm{P}$, Lecomte $\mathrm{F}$, et al. Liposome surface charge influence on skin penetration behaviour. Int J Pharm. 2011;411(12):223-231. doi:10.1016/j.ijpharm.2011.03.049

56. Zhou W, Liu W, Zou L, et al. Storage stability and skin permeation of vitamin C liposomes improved by pectin coating. Colloids Surf B Biointerfaces. 2014;117:330-337. doi:10.1016/j.colsurfb.2014.02. 036

57. Sala M, Diab R, Elaissari A, Fessi H. Lipid nanocarriers as skin drug delivery systems: properties, mechanisms of skin interactions and medical applications. Int $J$ Pharm. 2018;535(1-2):1-17. doi:10.10 16/j.ijpharm.2017.10.046

58. Alvarez-Roman R, Naik A, Kalia YN, Fessi H, Guy RH. Visualization of skin penetration using confocal laser scanning microscopy. Eur J Pharm Biopharm. 2004;58(2):301-316. doi:10.1016/j.ejpb.2004.03.027

59. Subongkot T, Wonglertnirant N, Songprakhon P, Rojanarata T, Opanasopit P, Ngawhirunpat T. Visualization of ultradeformable liposomes penetration pathways and their skin interaction by confocal laser scanning microscopy. Int J Pharm. 2013;441(1-2):151-161. doi:10.1016/j.ijpharm.2012.12.003

60. Chen-yu G, Chun-fen Y, Qi-lu L, et al. Development of a quercetin-loaded nanostructured lipid carrier formulation for topical delivery. Int J Pharm. 2012;430(1-2):292-298. doi:10.1016/j.ijpharm.2012.03.042

61. Foldvari M, Badea I, Wettig S, et al. Topical delivery of interferon alpha by biphasic vesicles: evidence for a novel nanopathway across the stratum corneum. Mol Pharm. 2010;7(3):751-762. doi:10.1021/ mp900283x

62. Kasetvatin C, Rujivipat S, Tiyaboonchai W. Combination of elastic liposomes and low frequency ultrasound for skin permeation enhancement of hyaluronic acid. Colloids Surf B Biointerfaces. 2015;135:458-464. doi:10.1016/j.colsurfb.2015.07.078

63. Freag MS, Elnaggar YS, Abdelmonsif DA, Abdallah OY. Layer-bylayer-coated lyotropic liquid crystalline nanoparticles for active tumor targeting of rapamycin. Nanomedicine. 2006;11(22):29752996. doi:10.2217/nnm-2016-0236

64. Noda M, Sugihara H, Tatsumi Y, et al. Effects of etodolac, a selective cyclooxygenase-2 inhibitor on the expression of E-cadherin-catenin complexes in gastrointestinal cell lines. $J$ Gastroenterol. 2002;37:896-904. doi:10.1007/s005350200151

65. Liang CC, Park AY, Guan JL. In vitro scratch assay: a convenient and inexpensive method for analysis of cell migration in vitro. Nat Protoc. 2007;2(2):329-333. doi:10.1038/nprot.2007.30

66. Ascione F, Caserta S, Guido S. The wound healing assay revisited: a transport phenomena approach. Chem Eng Sci. 2017;160:200-209. doi:10.1016/j.ces.2016.11.014

67. Tendo M, Yashiro M, Nakazawa K, et al. A synergic inhibitoryeffect of combination with selective cyclooxygenase-2 inhibitor and S-1 on the peritoneal metastasis for scirrhous gastric cancer cells. Cancer Lett. 2006;244(2):247-251. doi:10.1016/j.canlet.20 05.12.019

68. Kono M, Watanabe M, Abukawa H, Hasegawa O, Satomi T, Chikazu D. Cyclo-oxygenase-2 expression is associated with vascular endothelial growth factor $\mathrm{C}$ expression and lymph node metastasis in oral squamous cell carcinoma. J Oral Maxillofacial Surg. 2013;71 (10):1694-1702. doi:10.1016/j.joms.2013.04.015

69. Ozaslan KM, Kilic ID, Guldur IH, Emin M. Ehrlich ascites carcinoma. AfrJ Biotechnol. 2011;10(13):2375-2378. 
70. FEd S, Estrela A, Serakides GR, Cassali MG, Dantas G. Ehrlich tumor as model to study artificial hyperthyroidism influence on breast cancer. Pathol Res Pract. 2007;203(1):39-44. doi:10.1016/j.prp.2006.09.005

71. Habib SA, Aggour YA, Taha HA. Downregulation of transforming growth factor- $\beta$ (TGF- $\beta$ ) and vascular endothelial growth factor (VEGF) in ehrlich ascites carcinoma-bearing mice using stearic acid-grafted carboxymethyl chitosan (SA-CMC). Nat Sci. 2012;4 (11):808. doi: $10.4236 /$ ns.2012.411108
72. Rivenson A, Schnelle V, Moroson H, Madden R, Herp A. Variable response of spleen to ehrlich's tumor according to the physical form (ascitic or solid) of the tumor. Experientia. 1981;37(2):195-197. doi:10.1007/bf01963229
International Journal of Nanomedicine

\section{Publish your work in this journal}

The International Journal of Nanomedicine is an international, peerreviewed journal focusing on the application of nanotechnology in diagnostics, therapeutics, and drug delivery systems throughout the biomedical field. This journal is indexed on PubMed Central, MedLine, CAS, SciSearch ${ }^{\mathbb{B}}$, Current Contents ${ }^{\mathbb{B}} /$ Clinical Medicine, $^{2}$
Dovepress

Journal Citation Reports/Science Edition, EMBase, Scopus and the Elsevier Bibliographic databases. The manuscript management system is completely online and includes a very quick and fair peer-review system, which is all easy to use. Visit http://www.dovepress.com/ testimonials.php to read real quotes from published authors.

Submit your manuscript here: https://www.dovepress.com/international-journal-of-nanomedicine-journal 